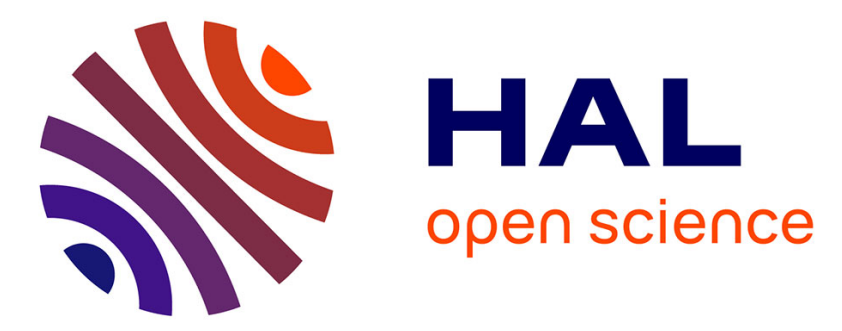

\title{
Patient-specific and real-time model of numerical simulation of the hemodynamics of type B aortic dissections
}

J Tomasi, F Le Bars, C Shao, A Lucas, M Lederlin, P Haigron, J P Verhoye

\section{- To cite this version:}

J Tomasi, F Le Bars, C Shao, A Lucas, M Lederlin, et al.. Patient-specific and real-time model of numerical simulation of the hemodynamics of type B aortic dissections. Medical Hypotheses, 2020, 135, pp.109477. 10.1016/j.mehy.2019.109477 . hal-02393818

HAL Id: hal-02393818 https://hal-univ-rennes1.archives-ouvertes.fr/hal-02393818

Submitted on 12 Feb 2020

HAL is a multi-disciplinary open access archive for the deposit and dissemination of scientific research documents, whether they are published or not. The documents may come from teaching and research institutions in France or abroad, or from public or private research centers.
L'archive ouverte pluridisciplinaire HAL, est destinée au dépôt et à la diffusion de documents scientifiques de niveau recherche, publiés ou non, émanant des établissements d'enseignement et de recherche français ou étrangers, des laboratoires publics ou privés. 


\title{
Patient-specific and real-time model of numerical simulation of the hemodynamics of type $B$ aortic dissections
}

\author{
J. TOMASI, MD ${ }^{1}$, F. LE BARS, MD ${ }^{1}, C_{.}$SHAO ${ }^{2}$, A. LUCAS, MD ${ }^{3}$, M. LEDERLIN, MD, PhD ${ }^{4}$, \\ P. HAIGRON, PhD ${ }^{5}$, J.P. VERHOYE, MD, PhD ${ }^{1}$
}

\author{
${ }^{1}$ Service de Chirurgie Thoracique et Cardiovasculaire, CHU Pontchaillou, Rennes, France \\ ${ }^{2}$ Ansys Inc., Villeurbanne, France \\ ${ }^{3}$ Service de Chirurgie Vasculaire, CHU Pontchaillou, Rennes, France \\ ${ }^{4}$ Service de Radiologie, CHU Pontchaillou, Rennes, France \\ ${ }^{5}$ Laboratoire Traitement du Signal et de L'image (LTSI), INSERM 1099U, Université Rennes 1, \\ Rennes, France
}

Corresponding author

Name: Florent LE BARS, MD

Address: CHU Pontchaillou

2 rue Henri Le Guilloux

35000 RENNES

FRANCE

E-mail: Florent.LE.BARS@chu-rennes.fr

Phone: 0033299285765

Fax: 0033299282496

\section{Funding}

The authors received no specific funding for this work, but it was made in partnership with Ansys Inc. 


\section{PREAMBLE}

This scientific research work is the fruit of the participation of a multidisciplinary team, involving doctors, researchers and engineers, confirmed and in the course of formation, within an association between the LTSI * and the Ansys Inc.**.

From this article follows the realization of a science thesis of a Doctor of Medicine, a doctorate of an engineer and a Master of Science of another Doctor of Medicine.

The role of the engineer consisted in the creation of the simulation model and the optimization of this one, whereas the role of the doctors of medicine was to allow a clinical coherence of the model during its development, to set up the clinical research allowing the acquisition and the collection of the medical data and the parameterization of the final model in order to recreate coherent clinical situations in simulation.

* Laboratoire Traitement du Signal et de l'Image, is an INSERM unit (Institut National de la Santé et de la Recherche Médicale), working in close collaboration with the University Hospital of Rennes on numerous medical research projects involving technologies and engineering sciences.

** American company specializing in Computational Fluid Dynamics (CFD) 


\begin{abstract}
Introduction: Regular monitoring of uncomplicated type B aortic dissection is essential because 25 to $30 \%$ will progress to aneurysmal form. The predictive factors of this evolution are not clearly defined, but they seem to be correlated with hemodynamic data.

Hypothesis: Our goal is to create a patient-specific and real-time model of numerical simulation of the hemodynamics of uncomplicated type B aortic dissections in order to predict the evolution of these pathologies for earlier treatment.

Method: This model consists in a coupling OD (hydraulic-electric analogy) - 3D (CT angiography segmentation) of the aortic arch with optimization by comparison to the 2D Phase Contrast MRI data and using Reduced Order Models to drastically reduce computing times. We tested our model on a healthy and a dissected patient. Then we realized different systolic blood pressure scenarios for each case, which we compared.
\end{abstract}

Results: In the dissected patient, the blood pressure at the false lumen wall was less important than the true lumen. Furthermore, the aortic wall shear stress and the velocity fields in aorta increase at the entry and re-entry tears between the two lumens. The simulation of different blood pressures scenarios shows a decrease in all these three parameters related to the decrease of the systolic blood pressure.

Conclusion: Our model provides reliable patient-specific and real-time 3D rendering. It has also allowed us to realize different flow variation scenarios to simulate different clinical conditions and to compare them. However, the model still needs improvement in view of a daily clinical application. 


\section{INTRODUCTION}

Aortic dissection is a rare (3.2 / 100,000 inhab. /year) but serious disease (50\% of mortality at 48 hours and $90 \%$ at 3 months), which realizes a cleavage within the aortic wall what creating a false lumen that may be responsible for aortic rupture and / or organ perfusion at the acute phase.

We distinguish the forms interesting the ascending aorta (type A dissection), where the risk of intra-pericardial rupture is high and for which urgent surgical management by replacement of the ascending aorta is the reference, and type B dissections which by definition does not interest the ascending aorta.

Currently, for the initial management of these type B aortic dissections, the decision tree is as follows:

- Uncomplicated dissections (i.e. without signs of rupture or mal-perfusion) should benefit from optimal medical treatment by blood pressure (BP) monitoring (systolic BP $<120 \mathrm{mmHg}$ ) and active medical supervision.

- Complicated dissections should be surgically treated, for which Thoracic EndoVascular Aortic Replacement (TEVAR) become the gold standard when anatomical conditions are favorable (1).

Two essential evolutionary phases of these initially uncomplicated dissections must be distinguished. The first is the acute phase between 0 and 30 days with a risk of mal-perfusion and rupture for which surgical intervention is associated in $24 \%$. The second is the chronic phase where 25 to $30 \%$ will evolve to aneurysmal form or secondary mal-perfusions at 4 years and will also have to benefit from a surgical procedure (2). 
The current clinical problem is: Whose propose an early endovascular procedure in uncomplicated situation to prevent chronic complications?

Existing anatomical elements that have been identified as predictors of poor long-term outcome in type B dissections, such as an initial aortic diameter over $40 \mathrm{~mm}$ in the acute phase, a false lumen over $22 \mathrm{~mm}$ of diameter, an elliptical true lumen and a circular false lumen and an entry tear located less than 5 centimeters from the origin of the left subclavian artery (3). But these are not enough to initiate a surgical management. The definition of this sub-population concerned is therefore still debated.

A better understanding of hemodynamic events such as comprehension of pressure regimes between the true and the false lumen, wall shear stress and blood velocities fields can help to refine this complex hemodynamics. Some of these data are accessible through medical imaging such as aortic geometry by CT or flow data by MRI. However, no examination can quantify the pressure and the shear stress that apply to the aortic wall, as well as the blood velocity fields. These data are therefore only accessible through computerized simulation models (Computational Fluid Dynamics (CFD)) that are currently rarely patientspecific and require a lot of computation time, which limits their clinical application. 


\section{HYPOTHESIS / GLOBAL PROBLEM}

The hypothesis of our work is to know if it is possible to predict the evolution of uncomplicated type B aortic dissections in time through numerical simulation of hemodynamics and this specifically for each patient.

One of the main challenges is to have shorter simulation times than real life so that the simulation tool can have a clinical interest. The ultimate interest is to determine profiles of patients at risk for chronic complications of their dissection and for whom earlier surgical management would avoid these complications.

\section{INITIAL PROBLEM}

The first step of the work consists in the realization of the patient-specific and real-time computer model, allowing the simulation of flow variations in the aortic arch in a healthy patient then in uncomplicated type B aortic dissections. 


\section{HYPOTHESIS ASSESMENT (Figure 1)}

\section{Clinical data management}

For the realization of our work we needed a healthy subject to create the healthy aorta model and a subject with uncomplicated type B aortic dissection to test the model. The healthy subject was included on the basis of volunteering and dissected subject was included prospectively in the acute phase of his pathology after information, written and oral, clear, fair and appropriate and not opposed from him to participate at the study. For both, the only inclusion criterion was to be major and the exclusion criteria were having a pathological aorta for the healthy subject, having aortic dissection of type A or type B complicated for the dissected subject, and present a contraindication to performing an MRI for both groups.

Clinical data such as sex, age, heart rate, blood pressure and treatments were also collected.

To carry out this study, we had the agreement of the patient protection committee through the protocol "hors loi Jardé" because it involves the human person but does not belong to an interventional study since the MRI is a common follow-up exam of the aortic dissections.

\section{Choice of OD mode}

To reduce computational time and make the model usable in clinical practice, we used aortic arch model reduction using a OD-3D coupled model to provide the boundary conditions for the aortic arch 3D simulation. This OD model is based on the concept of hydraulic-electrical analogy: blood flow equivalent to an electrical current, blood pressure between two points to a voltage, vessel elasticity to a capacitance (C), vascular resistances to a resistance (R) and blood inertia to an inductance (L). This analogy is based on the assumption that the 
physiological variables of interest (e.g. pressure, flow and volumes) are uniformly distributed in space. This model works in a closed-loop to get as close as possible to the full cardiovascular system (CVS) with its three compartments, which are the systemic circulation, the pulmonary circulation and the heart. After a review of the literature we chose the OD model of Korakianitis et al. (4) because it is a simple model that represents the full CVS in the form of closed loop while integrating various vascular segments of interest (coronary sinuses, arteries, arterioles, capillary, veins). We then reproduced it on the Twin Builder software (Ansys, Canonsburg, Pennsylvania, USA) (Figure 2).

\section{D geometrical modeling of the aorta}

In our work we included two patients, one with healthy aorta and one with dissected aorta (uncomplicated type B dissection starting just after the ostium of the left subclavian artery at the outer aortic arch curvature) having already been operated several years ago on a mechanical Bentall procedure (replacement of the aortic valve with a mechanical prosthesis and of the initial part of the aorta with a synthetic tube) for aortic root aneurysm with aortic valve regurgitation.

In these two patients we performed a chest CT scan, at low X-ray dose and without contrast injection for the patient with healthy aorta and normal X-ray dose and contrast injection for the dissected patient. These scanners made it possible to perform aortic segmentation, using 3DSlicer software (open source software), to obtain the 3D geometric models of these aortas (Figure 3). At the same time, we realized a 2D Phase Contrast (PC) MRI, to obtain noninvasively the hemodynamic data that will serve as a reference for the confrontation of our model. 
For a sake of simplicity to create our model, we were interested only at the hemodynamic study of the aortic arch and the first centimeters of the descending thoracic aorta (abandonment of the ascending aorta (AA) because it does not correspond to the definition of dissection of type B). This gives us a system with one inlet (initial part of the arch) and four outlets (brachiocephalic trunk (BT), left common carotid artery (LCC), left subclavian artery (LS) and descending thoracic aorta (DA)) for the healthy patient and five outlets (BT, LCC, LS, true and false lumen (TL and FL)) for the dissected patient. Dissected patient to whom we also found two entries tears of the dissection just after the ostium of the LS, and two re-entries in the DA.

\section{OD Model modification}

The direct coupling between the OD model of the full CVS and the 3D aortic geometry is complicated because the values of the OD model parameters of the CVS are not available in the literature. This step is essential because a wrong calibration of its parameters can lead to a problem of correlation of the output of the 3D model of the stick which produces numerical instabilities.

To circumvent this problem, an equivalent OD model of aortic arch was created, based on static simulations (absence of the time effect, open circuit) and electric model (inductance) to have an approximation of the 3D transient fluid simulation of the arch and for secondarily be coupled to the OD model of the full CVS. Then this model allowed us to obtain pressure and flow curves that served us as boundary conditions for our 3D simulation.

3D models are based on the resolution of Navier-Stokes equations, applied to the finite element discretization of the 3D model. The 3D model of aortic arch was divided into five or six segments depending on the patient to represent each inlet and outlets branches. Each 
segment of the aortic arch was represented using an inductance and a pressure source whose values are derived from static simulations. In our models we make the assumption that the wall is rigid, so we did not use capacitance. For the representation of the wall of the aortic arch the 3D volume consisted of a polyhedral mesh and eight prism layers, for the blood a Newtonian incompressible fluid model was used and for the model of turbulence the model SST k- $\omega$.

For the computation of the pressures, the static simulation consists of applying an inlet pressure (Pinlet) at the ascending aorta and flow rates at each outlet (Qoutlet) in order to determine the outlet pressures (Poutlet). For this we did this simulation many times by varying the values of Pinlet and Qoutlet applied. We have recovered at each calculation calculated Poutlet (static ROM). From the results, we used a response surface which made it possible to reduce the calculation times. This makes it possible to obtain instantaneously the values of the pressure sources for each set of boundary conditions. For the calculation of the inductance parameter in each branch, we used the geometrical values of each branch (radius and length) assuming it was a perfect cylinder and we applied the Poiseuille law (Figure 4).

Then, the OD model based on static simulations of the aortic arch was coupled to the full CVS model. The three upper outlets were connected to a block consisting of resistance, capacitance and inductance to represent the systemic arterioles, capillaries and veins of the upper body. The two lower outlets were connected to the same block type to represent the systemic part of the lower body. The fact to having capacitances in the full CVS model makes it possible to simulate the compliance of the vessels. As a result, in our full CVS model, only the aortic arch is modeled by a rigid wall. 
The integration of the static model of the aortic arch with the OD model of the full CVS allowed us at this stage to have a full CVS OD model with the representation of the aortic arch (Figure 5).

\section{Personalization of the OD Model using the 2D PC MRI Flow Data}

Once the basic model OD with integration of the aortic arch was realized, it remained to calibrate it to make it patient-specific. The adjusted parameters were the resistance, capacitance, and inductance values of the systemic part of the model, as well as the atrial and ventricular elastance parameters. The aim was to correlate the flows at the outlet calculated from the OD model (flow / pressure curves - Dynarom) to the flows obtained from the patient's 2D PC MRI data, which serve as objective references. And this throughout the cardiac cycle, in systole and diastole.

For this we used the Levenberg-Marquardt optimization method to optimize the model (5). This method solves non-linear least squares problems and has shown good performance in minimizing least-squares curve fitting.

As a result of the optimization, the flows rates at the outlet of the $3 \mathrm{D}$ transient fluid simulations and the OD model of the full CVS were very close, particularly in the systolic phase. Note that the reverse flow phenomenon is underestimated with the OD model for all outputs, except for true light. (Figure 6).

\section{OD model simulation results (flow / pressure curves)}

Once the OD model was set up, we performed the simulations using the Twin Builder software to obtain the results of the simulation in the form of flow and pressure curves (pulsatile blood flow) (Figure 7). 


\section{Attribution of the OD model's boundary conditions}

However, all the difficulty in this OD model was the attribution of the boundary conditions so that the results from the $3 \mathrm{D}$ transient fluid simulation were clinically plausible. We performed several calculations with different boundary conditions to obtain multiple results in order to create a Reduced-Order Modeling (ROM). As a reminder, a ROM aims to reduce the complexity of mathematical calculations by reducing the size of the space or the degrees of freedom to obtain an approximation of the original model. The ROM requires an important learning set, hence the interest to having a wide range of variation between the different boundary conditions obtained. Value ranges are then assigned for the input flow and the outputs pressures (e.g. PAs [80; $180 \mathrm{mmHg}]$ ).

We also varied the parameters of the OD models (resistances, capacitances, inductance and elastance) to obtain different ranges of values for the input flow and the pressure. For each set of boundary conditions, we performed a 3D transient fluid simulation for which the computation time is approximately 24 hours for 3 cardiac cycles.

\section{Integration of data from the OD model and 3D representation}

To obtain the images of the simulation, at each calculation, we saved the values that interested us at each time step chosen. In our study we are interested only in the peak systole to study the part of the cardiac cycle where the blood pressure is the most important. On the other hand, for ROM and 3D calculations we took into account the whole cardiac cycle.

From these results we could create ROMs. Our ROM model consists of nonlinear differential equations that connect the solver inputs to the solver outputs using neural network methods. It provides a real-time approximation of the results of the physical solver (versus $24 \mathrm{~h}$ for 3 cardiac cycles for 3D transient fluid simulation). 
At this stage of our work we use two different types of ROMs: one calculates the transient results of aortic flow and pressure to replace the static model (dynamic ROM) and the other calculates the 3D physical variables (dynamic ROMs) which provides 3D values of the aortic arch for each of the hemodynamic data of interest $(x 3)$ : wall shear stress, wall pressure and blood velocity field.

The results obtained from the 3D dynamic ROM simulation were then transmitted to the CFD-Post software (Ansys) to obtain the 3D representations and make comparisons between the different scenarios (Figure 8).

\section{Model optimization}

Once the aortic arch dynamic ROM was functional, it replaced the initial static OD model of the aortic arch (static simulations of pressures and inertances) to obtain a OD dynamic ROM model of the aortic arch.

Parameters of the OD model (dynamic this time) were optimized by a new confrontation with the 2D PC MRI data to match to the clinical data of the patient at rest.

The comparison between our final OD dynamic ROM model and the 3D transient fluid simulation shows a relative difference on average $1.55 \%$ and an absolute maximum difference of $4.26 \mathrm{mmHg}$ for all cumulative Poutlets for the healthy patient. For the dissected patient, these values are $0.61 \%$ and $3.66 \mathrm{mmHg}$ respectively. Knowing that the computation time of the 3D transient fluid simulations is on average 17 hours for the healthy patient and 23 hours for the dissected patient, while the OD dynamic ROM model only takes a few seconds for both cases. 


\section{Use in a clinical situation}

Now we have a final OD model with integration of the aortic arch for a healthy patient and for a dissected patient (model OD dynamic ROM of the arc and model OD full CVS) (Figure 9). This allows us to perform different flow simulations by modifying certain parameters, such as heart rate, to simulate certain real-life situations (stress, stress) or the effects of a medication (antihypertensive, bradycardic) and obtain hemodynamic data from the aortic arch in real time.

\section{EMPIRICAL DATA}

In our research, we had two patients, one with a healthy aorta and the other with a dissected aorta, with whom we wanted to simulate different scenarios of BP variations in order to test our model and then be able to consider different scenarios of prediction of evolution of type B aortic dissection as a function of adherence to medical treatment.

For the healthy patient with controlled systolic BP (sBP) (i.e. sBP $<120 \mathrm{mmHg}$ ) measured at $115 \mathrm{mmHg}$ and diastolic BP (dBP) at $75 \mathrm{mmHg}$ on 2D PC MRI, we simulated high blood pressure (HBP) (i.e. sBP > $140 \mathrm{mmHg}$ ).

For the dissected patient with moderately controlled sBP (i.e. sBP between 120 and 140 $\mathrm{mmHg}$ ) measured at $135 \mathrm{mmHg}$ and $\mathrm{dBP}$ at $73 \mathrm{mmHg}$ on 2D PC MRI, we used two scenarios of $\mathrm{sBP}$. A reduction to obtain a controlled sBP (i.e. $<120 \mathrm{mmHg}$ ) and an increase to obtain an uncontrolled sBP (i.e. > $140 \mathrm{mmHg}$ ).

As a reminder, in the case of aortic dissections, the blood pressure objective is less than $120 \mathrm{mmHg}$. 


\section{Healthy patient case}

\section{"High pressure" scenario}

To simulate the "high pressure" scenario, we chose to simulate vasoconstriction by increasing vascular resistance (venous) without changing the cardiac frequency (CF).

Before simulation and according to the MRI data the healthy patient had a systolic ejection volume (SEV) at $93 \mathrm{ml}$ and a CF at $66 \mathrm{bpm}$, which by the relation $\mathrm{Q}=\mathrm{SEV} \times \mathrm{CF}$ gave a cardiac flow (Q) at $6.1 \mathrm{~L} / \mathrm{min}$ and a $\mathrm{Q}$ peak at $0.45 \mathrm{~L} / \mathrm{sec}$.

After simulation through our OD model we achieved HBP with a sBP at $145 \mathrm{mmHg}$ with an increase of dBP at $115 \mathrm{mmHg}$ but a decrease of $\mathrm{Q}$ peak at $0.38 \mathrm{~L} / \mathrm{sec}$.

\section{Healthy patient simulations}

A comparison of the aortic wall pressure shows a greater wall pressure in the "high pressure" scenario. It is also noted that the blood flow is laminar in both cases since there is very little pressure wall variation (Figure 10).

The comparison of the aortic wall shear stress shows more stress zones at the level of the Supra-Aortic Trunks (SAT) but not at the level of the aorta. The analysis of the differences between the two models shows a very small difference in the order of less than $20 \mathrm{~Pa} / 0,15$ $\mathrm{mmHg}$ in favor of greater stress for the healthy patient with "controlled pressure" scenario (Figure 11).

The comparison of the aortic blood velocities fields shows accelerations at the level of the SAT and at the outer curvature of the initial part of the aortic arch. The analysis of the differences between the two models shows a very small difference of the order of less than $0.5 \mathrm{~m} / \mathrm{sec}$, which is also greater for the "controlled pressure" scenario (Figure 12). 


\section{Dissected patient case}

\section{"Controlled pressure" scenario}

To simulate the "controlled pressure" scenario, we chose to simulate beta-blocker antihypertensive drug use as it is the main antihypertensive drug used in aortic dissection. The action of beta-blockers is primarily at the cardiac level by reducing heart rate, cardiac excitability and myocardial contractibility. So, we focused on acting on these parameters through our model, to reduce cardiac output. Based on the patient's MRI data, the patient had a SEV at $69 \mathrm{ml}$, a CF at $76 \mathrm{bpm}$, a Q at $5.3 \mathrm{~L} / \mathrm{min}$ and a $\mathrm{Q}$ peak at $0.38 \mathrm{~L} / \mathrm{sec}$. We therefore first directly decreased the heart rate at $60 \mathrm{bpm}$ in the parameters of our model and secondly divided by 8.33 the initially defined parameters of elastance of the left atrium and the left ventricle to have a SEV equivalent to $69 \mathrm{ml}$. With these two modifications we obtained through the OD simulation a $Q$ at $4.1 \mathrm{~L} / \min (69 \times 60 / 1000)$ with a $Q$ peak at $0.31 \mathrm{~L} / \mathrm{sec}$, a sBP peak at $112 \mathrm{mmHg}$ and a dBP peak at $55 \mathrm{mmHg}$.

\section{"High pressure" scenario}

To simulate the "high pressure" scenario we had several physiological possibilities of BP increase. We chose to vary the responsible factors of chronic HBP, either arterial resistance simulating vasoconstriction or venous capacitance simulating hypervolemia (7).

First, note that the sum of systemic vascular resistance in our OD model for the dissected reference patient is $82 \mathrm{MPa} . \mathrm{s} / \mathrm{m} 3$ for normal values from the literature (6) between 70 and 160 MPa.s / m3 reinforcing the accuracy of our model. Moreover, in our OD model, for the reference dissected patient the arterial resistances of the FL are less important than those of the TL. 
In both "vasoconstriction" and "hypervolemia" scenarios we were able to obtain the same $\mathrm{SBP}$ values of $165 \mathrm{mmHg}$, either by increasing the arterial resistances 6.5 times, or by increasing the venous capacitance 2.5 times. On the other hand, the values of $d B P$ and $Q$ differed. In the "vasoconstriction" scenario, the $\mathrm{dBP}$ was $92 \mathrm{mmHg}$ and the Q peak was stable at $0.37 \mathrm{~L} / \mathrm{sec}$ for a stable $\mathrm{Q}$ as well. In the "hypervolemia" scenario, the $\mathrm{dBP}$ was $88 \mathrm{mmHg}$, whereas the Q peak was increased to $0.47 \mathrm{~L} / \mathrm{sec}$, indicating an increase in $\mathrm{Q}$.

\section{Dissected patient simulations}

The comparison of the aortic wall pressures of the 4 scenarios applied shows for each of the scenarios a lesser pressure at the FL wall (approximately $30 \mathrm{mmHg}$ ) (Figure 13). As for the $\mathrm{TL}$, the wall pressure of the $\mathrm{FL}$ also increases with the increase of sBP. For the last two scenarios (Figure $13 \mathrm{C}$ and $\mathrm{D}$ ) the pressure in the arch and the TL is broadly similar, however the $Q$ peak causes a slight increase of the pressure wall of the FL for the scenario "high pressure by hypervolemia" (Figure $13 \mathrm{D}$ ).

The comparison of the aortic wall shear stress of the 4 scenarios applied shows for each of the scenarios, zones of greater wall shear stress at the level of the suture zone of the ascending aorta, at the two entries tears of the dissection after the ostium of the left subclavian artery, at the two re-entries tears in the descending thoracic aorta and at the aortic wall of the FL in front these four communications (Figure 14). It is also noted that these wall shear stress increase at these locations with increasing SBP. As for wall pressures, the wall shear stress at the aortic wall are increased in the "high pressure by hypervolemia" scenario (Figure $14 \mathrm{D}$ ) where the $\mathrm{Q}$ peak is higher.

The comparison of aortic blood velocities fields of the 4 scenarios shows for each of the scenarios, the accelerations of blood velocities at the level of the suture zone of the ascending 
aorta, at the two entries tears of dissection after leaving the ostium of the left subclavian artery and the two re-entries in the descending thoracic aorta (Figure 15). We also note that these speeds increase at these locations with the increase in SBP. As with wall pressures and wall shear stress, blood velocities in the aorta are increased in the "high pressure by hypervolemia" scenario (Figure $15 \mathrm{D}$ ) where the Q peak is higher.

\section{DISCUSSION}

\section{Results interpretation}

The analysis between the two scenarios in the healthy patient, shows a very small difference in values, of the order of less than $20 \mathrm{~Pa} / 0,15 \mathrm{mmHg}$ for wall shear stress and less than $0.5 \mathrm{~m} / \mathrm{sec}$ for blood velocities, favor of the "controlled pressure" scenario. This is explained by the fact that the $Q$ peak of the patient "controlled pressure" is greater ( $0.45 \mathrm{vs}$ $0.38 \mathrm{~L} / \mathrm{sec}$ ). This notion shows the interest of decreasing sBP but also $\mathrm{Q}$ in the management of patients in the context of cardiovascular prevention (role of betablockers).

In the dissected patient, analysis of the results showed a decrease of wall pressure in the FL and a decrease of wall shear stress and blood velocities at the level of communications between the two lumens related to the decrease in SBP. This confirms the interest of a reduction of SBP in the management of aortic dissections to limit the extension of the FL and its rupture in the acute phase and allow its healing and to limit the risk of aneurysmal evolution (main complication of aortic type B dissections in long-term) $(8,9)$.

We also note that vascular sutures disrupt vessel architecture and hemodynamics, making them areas of weakness, even at a distance from surgery. 


\section{State of art}

The first step in our work through this article is a feasibility study of our patient-specific and real-time fluid model of numerical simulation. The findings found are similar to other studies simulating aortic flow by fluid mechanics in uncomplicated type B aortic dissections (10-15). They also find an increase of wall shear stress at the entries and re-entries associated with an increase in velocity fields at the same level. This confirms the good functioning of our model. On the other hand, these models only transcribe numerically the clinical data of these patients and do not allow the modification of characteristics such as BP or Q to simulate other hemodynamic conditions.

Alimohammadi et al. (14) and Dillon-Murphy et al. (15) also integrate a OD model but use a Windkessel OD model which is an open model unlike our closed loop of the full CVS model which allows us a more precise description of the blood circulation, and therefore to make different scenarios by varying the parameters, which is much more restricted with a Windkessel model because of the absence of compartmentalization of the systemic circulation.

Moreover, thanks to the use of the ROMs we can obtain the results in real time contrary to the 3D transient fluid simulations ( $24 \mathrm{~h}$ for 3 cardiac cycles).

At this stage of our work the originality of our model resides in the way whose is obtained our model of simulation through a full CVS OD model and ROM, offering multiple technical and clinical advantages. 


\section{Advantage of OD models (full CVS, static arch + inductance)}

As previously stated, our OD model is closed (full body) which allows to have a correlation between the inlet and outlet and thus a better numerical stability, allowing the realization of different scenarios.

Moreover, the model integrates many compartments (heart, aortic arch, capillary arterioles, veins, ...), this allows to simulate different clinical scenarios by varying the parameters of the model and at different levels.

As for him, the model OD static + inductance of the arch, makes it possible to avoid directly coupling of the full CVS OD model with the 3D model and to obtain pressure and velocity curves for the 3D computation which is more stable numerically for the calculation.

\section{ROM innovations}

The ROM allows to have an instant and realistic representation of the aortic arch in the OD dynamic ROM model. This makes the optimization process possible since the number of calculations is important.

The ROM also allows to have 3D results for any setting of the OD system and this very quickly (few seconds).

\section{Advantage of our models}

The advantages of any numerical flow simulation reside in obtaining hemodynamic data in a non-invasive way for patients and at lower cost.

The advantage of the ROM compared to the 3D transient fluid simulation is that the computation time is drastically reduced from one day to a few seconds for a result difference 
less than $2 \%(1.55 \%$ and $0.61 \%)$. This temporal advantage (real-time) makes it possible to envisage a daily clinical application which was until now impossible.

Due to the use of CT images for the 3D geometrical representation of aortas coupled with hemodynamic data by the realization of a 2D PC MRI, we were able to create a patient specific simulation model, which allows have applicable results to each patient in the daily clinic.

The advantage of the OD model is that it makes it possible to interact with the various characteristics of the model in order to vary different values such as CF, Q and / or BP and thus perform 3D flow simulations that are not derived from the measurements of the MRI data of the patient but derived from hypothetical scenarios while remaining patient specific.

\section{Limitations}

There are several limits to our work. Firstly, we have been limited to the study of the aortic arch and not to the entire aorta for reasons of simplicity of the model. We have imposed to the ROM of the aortic arch a rigid and not compliant wall (unlike the rest of the OD model of the full CVS) in order to make fewer complex calculations. We have only confronted our model to one healthy patient and one dissected patient. To obtain the ROMs that allow instantaneous results, it is necessary first to have made several 3D fluid calculations, which takes time (about 24 hours). The use of a 4D PC MRI would allow a better confrontation of the results obtained. There is also a bias that emerges from the fact that the dissected patient has already undergone cardiac surgery of the aortic valve and the initial segment of the ascending aorta, which can disturb the hemodynamics at the exit of the heart.

As this work is still in the research phase, several steps are not yet optimized for an instant clinical application. It requires four different computing platforms and manual controls (OD model setting, encoding of commands, modification of extensions). 


\section{Expected improvements}

This work presented being that the first part of a research work more consequent, the model is destined to evolve. The first step will be to create a model of full aorta, from the aortic sinus to the iliac arteries with integration of a compliant wall of this aorta.

The second step will be the integration of a fatigue model of the aortic wall to simulate several days or months of cardiac cycles to obtain a predictive model of evolution of the aortic dissections specific to each patient. The difficulty is to obtain less computation time than the real time. This would determine the patients most at risk of developing aneurysms and progression of dissections. The goal is to provide earlier treatment of uncomplicated type B aortic dissections.

\section{CONCLUSION}

The use of the OD model and ROMs enables a reliable patient-specific and real-time numerical simulation of the hemodynamics of uncomplicated type B aortic dissections, opening the doors to clinical use. They also allow simulations in different flow conditions, which allowed us to confirm the interest of blood pressure reduction in the treatment of aortic dissections. Being at the beginning of our research, the model is destined to evolve, the goal is to predict the long-term evolution of aortic dissections and to offer earlier treatment to patients. 


\section{REFERENCES}

1. Olsson C, Thelin S, Ståhle E, Ekbom A, Granath F. Thoracic aortic aneurysm and dissection: increasing prevalence and improved outcomes reported in a nationwide population-based study of more than 14,000 cases from 1987 to 2002. Circulation. 12 déc 2006;114(24):2611-8.

2. Weiss G, Wolner I, Folkmann S, Sodeck G, Schmidli J, Grabenwöger M, et al. The location of the primary entry tear in acute type B aortic dissection affects early outcome. Eur J Cardiothorac Surg. sept 2012;42(3):571-6.

3. Booher AM, Isselbacher EM, Nienaber CA, Trimarchi S, Evangelista A, Montgomery DG, et al.The IRAD classification system for characterizing survival after aortic dissection. Am J Med. août 2013;126(8):730.e19-24.

4. Korakianitis T, Shi Y. A concentrated parameter model for the human cardiovascular system including heart valve dynamics and atrioventricular interaction. Medical Engineering \& Physics 28.7 (sept. 2006).

p. 613-628, ISSN : 1350-4533, DOI : 10.1016/j.medengphy.2005.10.004.

5. Gavin HP. The Levenberg-Marquardt method for nonlinear least squares curve-fitting problems. In 2013.

6. Washington University School of Medecine Department of Surgery. Klingensmith, Mary E. Li Ern Chen; Sean C Glasgow (2008).

7. G. D. Fink, «Sympathetic Activity, Vascular Capacitance and Long-Term Regulation of Arterial Pressure », Hypertension, vol. 53, no 2, p. 307-312, févr. 2009.

8. Editor's Choice - Management of Descending Thoracic Aorta Diseases - European Journal of Vascular and Endovascular Surgery. 
9. Erbel R, Aboyans V, Boileau C, Bossone E, Bartolomeo RD, Eggebrecht H, et al. 2014 ESC Guidelines on the diagnosis and treatment of aortic diseasesDocument covering acute and chronic aortic diseases of the thoracic and abdominal aorta of the adultThe Task Force for the Diagnosis and Treatment of Aortic Diseases of the European Society of Cardiology (ESC). Eur Heart J. 2014 Nov 1;35(41):2873-926.

10. Shang EK, Nathan DP, Fairman RM, Bavaria JE, Gorman RC, Gorman JH, et al. Use of computational fluid dynamics studies in predicting aneurysmal degeneration of acute type B aortic dissections. Journal of Vascular Surgery. 2015 Aug;62(2):279-84.

11. Cheng Z, Tan FP, Riga CV, Bicknell CD, Hamady MS, Gibbs RG, et al. Analysis of flow patterns in a patient-specific aortic dissection model. J Biomech Eng 2010;132:051007.

12. Karmonik C, Partovi S, Muller-Eschner M, Bismuth J, Davies MG, Shah DJ, et al. Longitudinal computational fluid dynamics study of aneurysmal dilatation in chronic DeBakey type III aortic dissection. J Vasc Surg 2012;56:260-3.

13. Cheng Z, Riga C, Chan J, Hamady M, Wood NB, Chesire JW, et al. Initial findings and potential applicability of computational simulation of the aorta in acute type B dissection. J Vasc Surg 2013;57(Suppl): 35S-45S.

14. Alimohammadi M, Agu O, Balabani S, Díaz-Zuccarini V. Development of a patient-specific simulation tool to analyse aortic dissections: Assessment of mixed patient-specific flow and pressure boundary conditions. Medical Engineering \& Physics. 2014 Mar;36(3):27584.

15. Dillon-Murphy D, Noorani A, Nordsletten D, Figueroa CA. Multi-modality image-based computational analysis of haemodynamics in aortic dissection. Biomechanics and Modeling in Mechanobiology. 2016 Aug;15(4):857-76. 
FIGURES

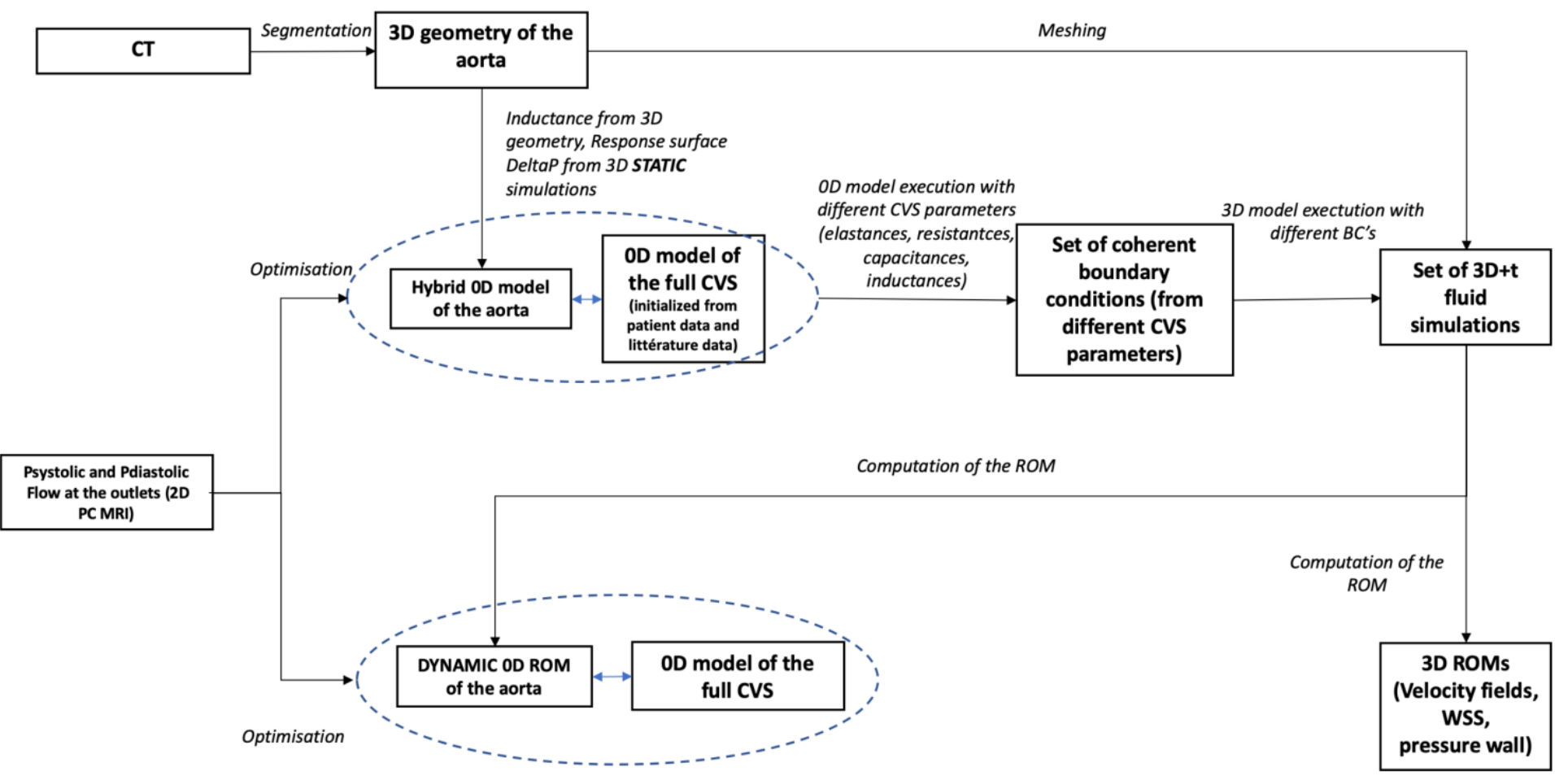

Figure 1: Global diagram of the realization of the simulation model

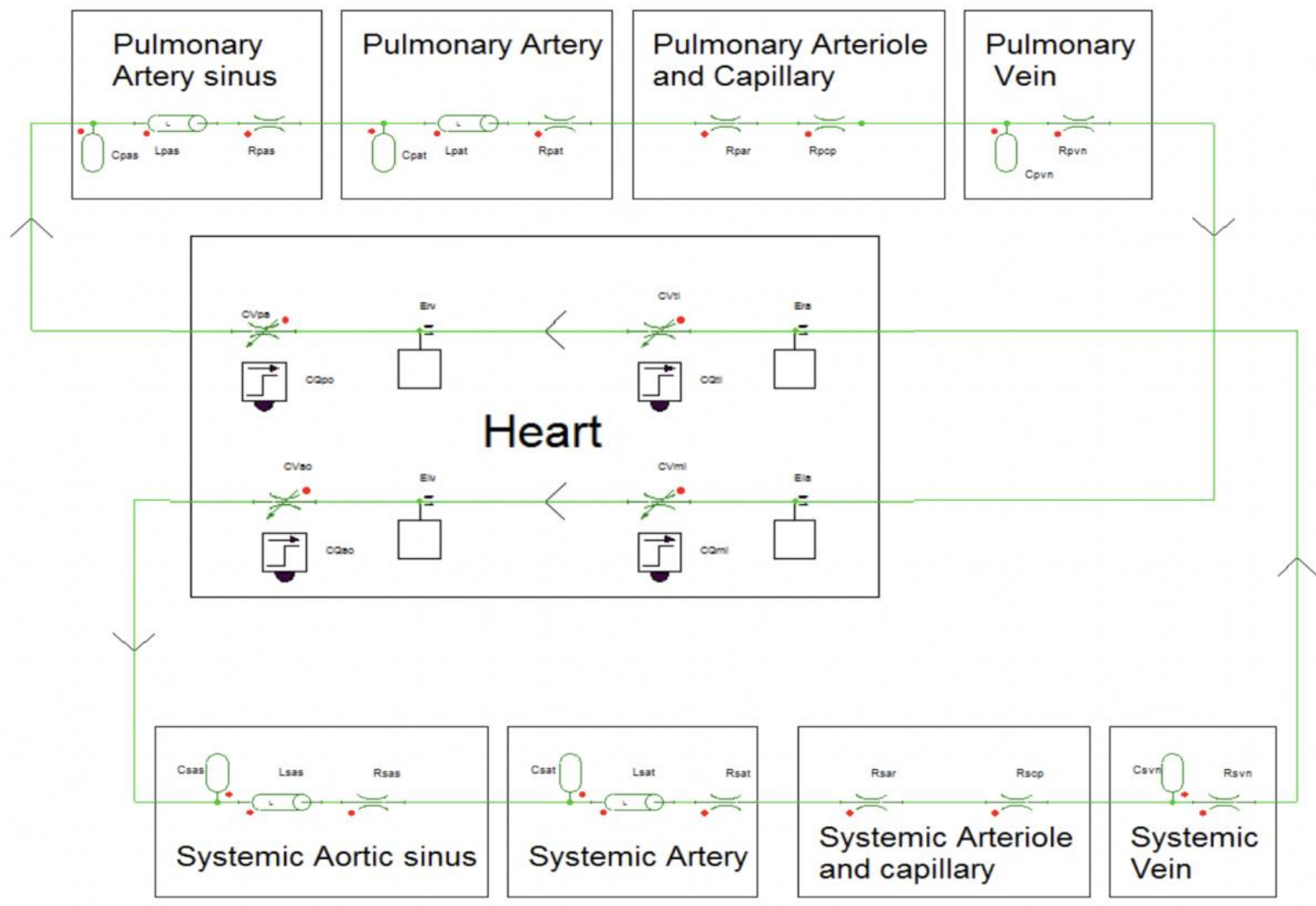

Figure 2: OD representation of the cardiovascular system 

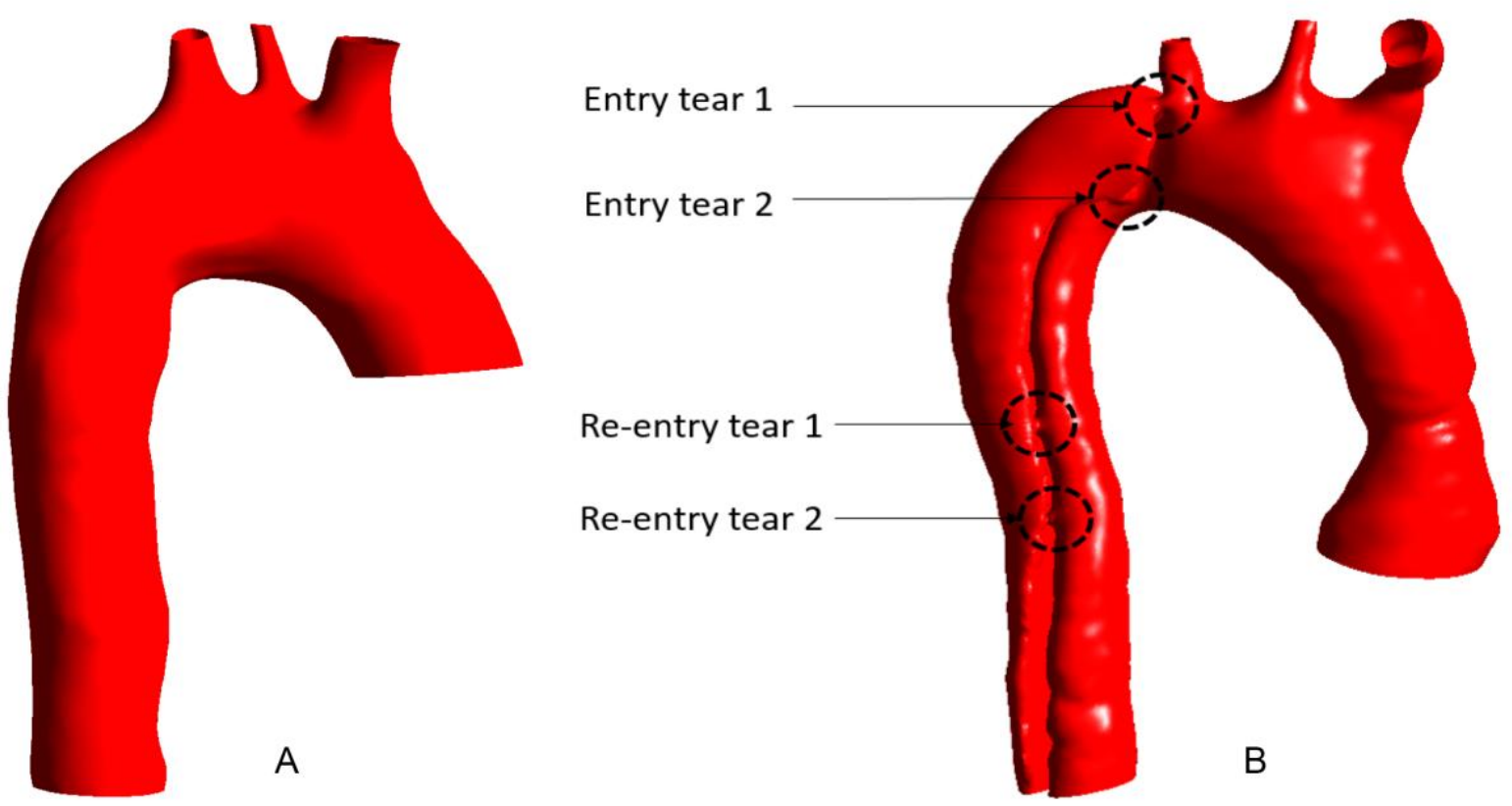

Figure 3: Surface rendering of aortic arch of healthy (A) and dissected (B) patient after segmentation
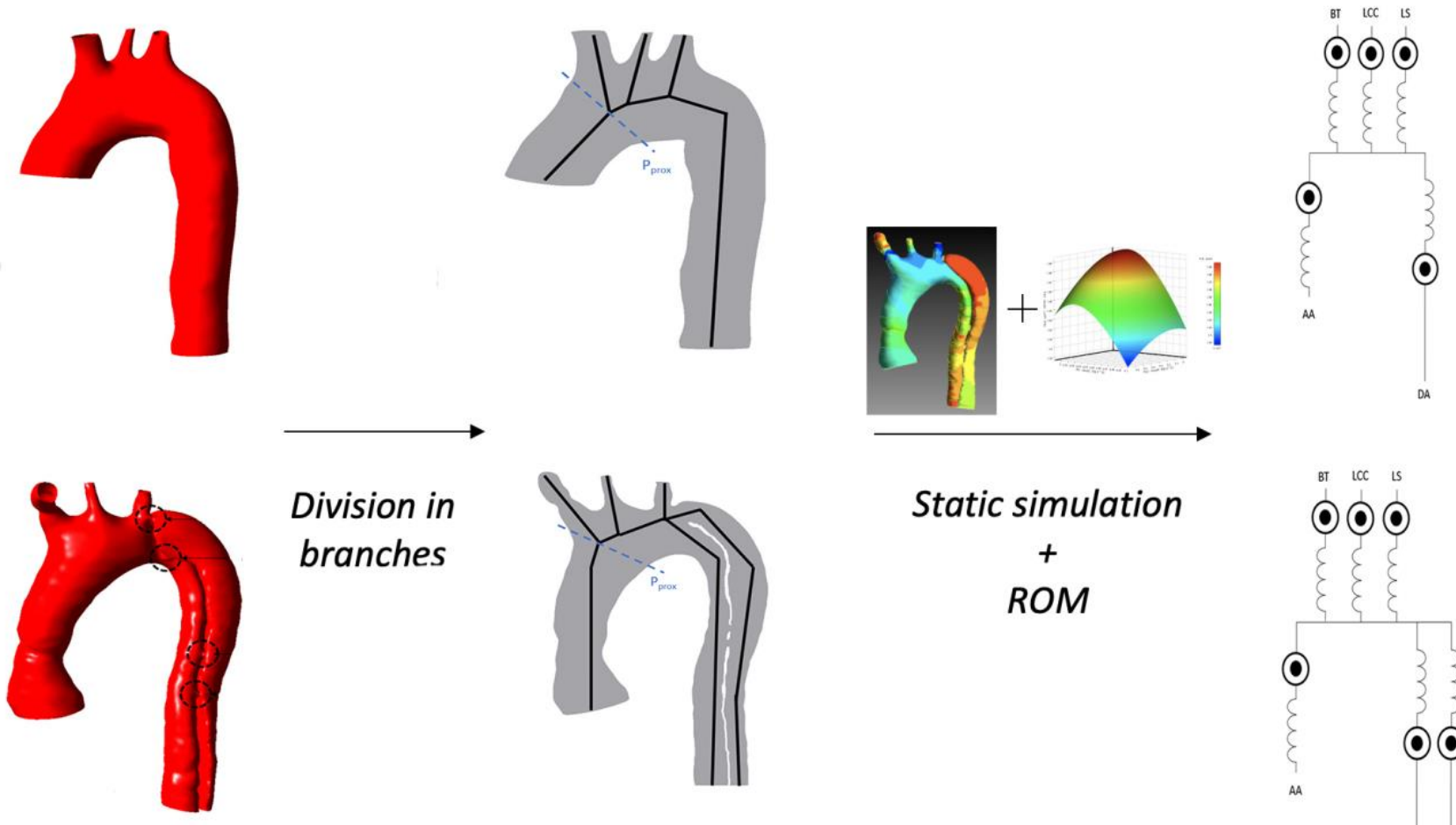

\section{Division in branches}

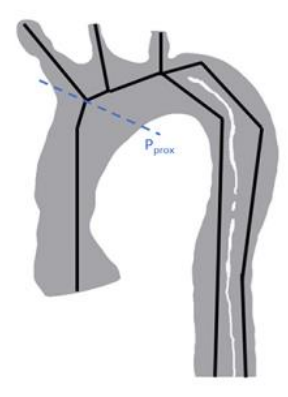

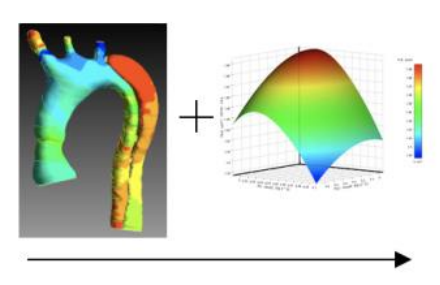

Static simulation

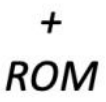

Figure 4: Steps to obtain the OD model of static simulation of the aortic arch for each patient 


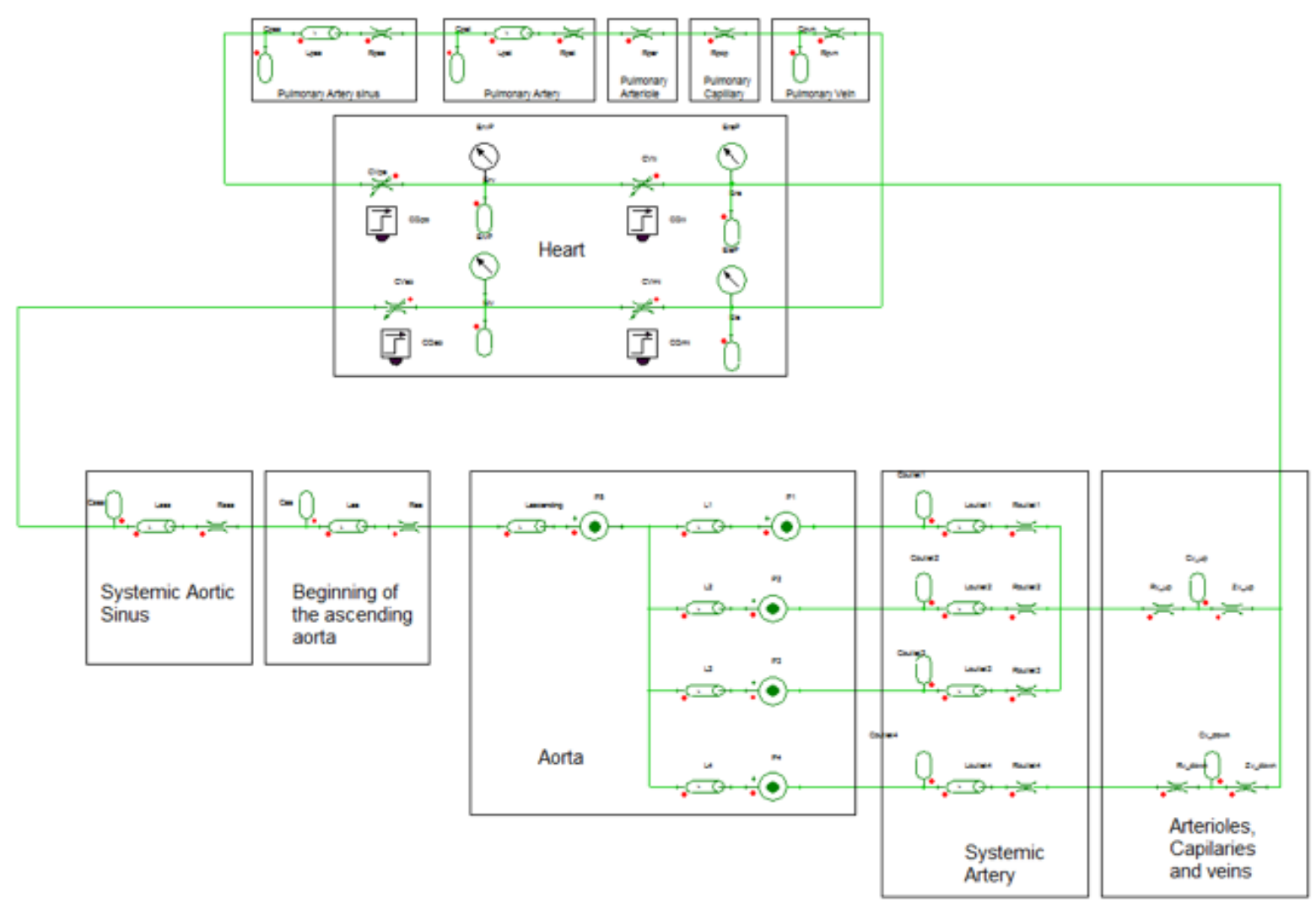

Figure 5: Full cardiovascular system OD model with integration of the static model of the aortic arch of the healthy patient 


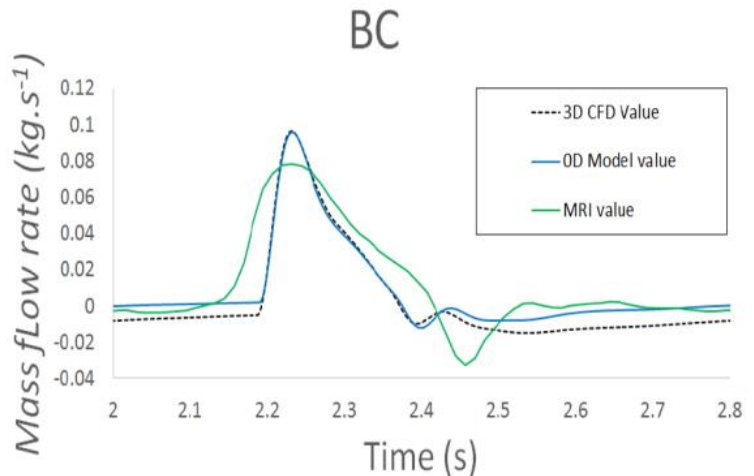

A

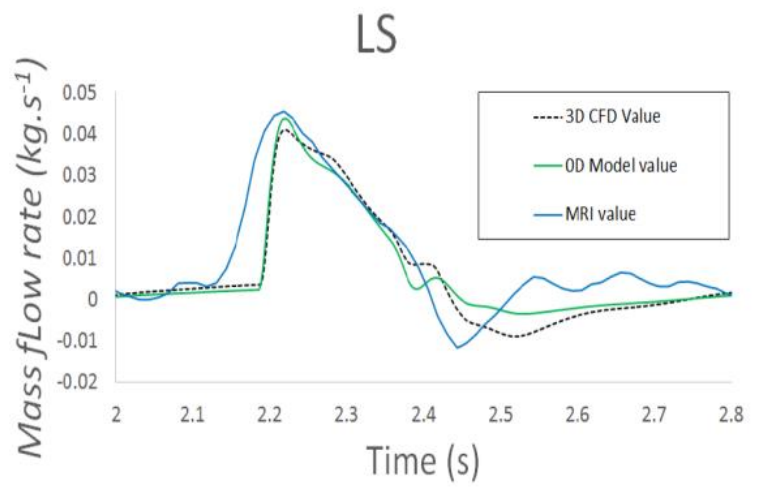

C

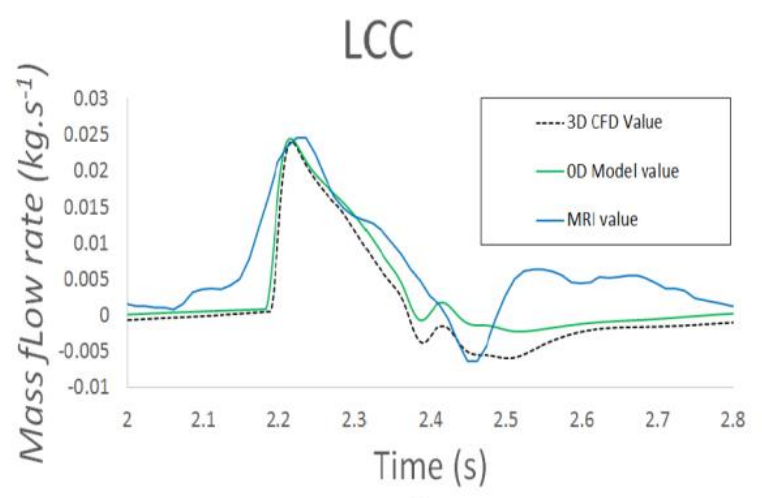

B

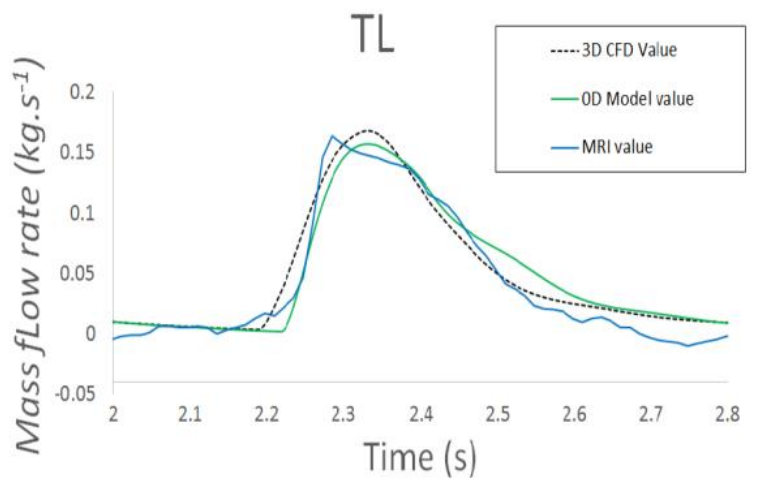

$\mathrm{D}$

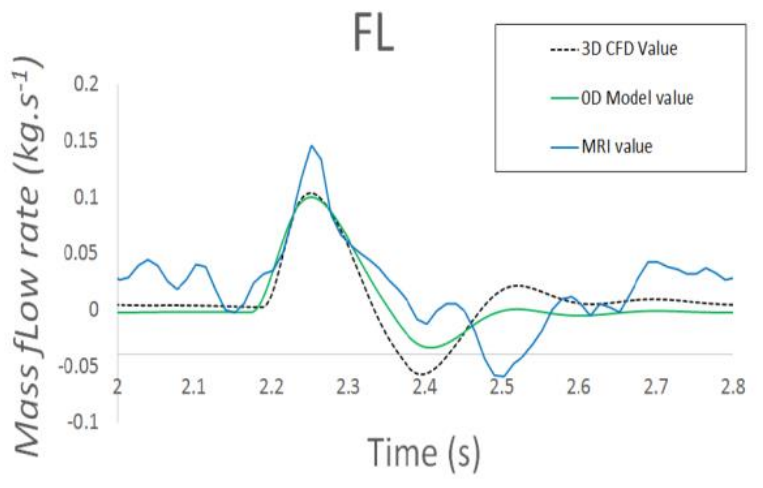

$\mathrm{E}$

Figure 6: Comparison of the flow curves at each outlet between the results of the 3D transient fluid and OD simulations, and the values of the 2D PC MRI

A: Flow in the Brachiocephalic Trunk (BT); B: Flow in the Left Common Carotid (LCC); C: Flow in the Left Subclavian (LS); D: Flow in the True Lumen (TL); E: Flow in the False Lumen (FL) 


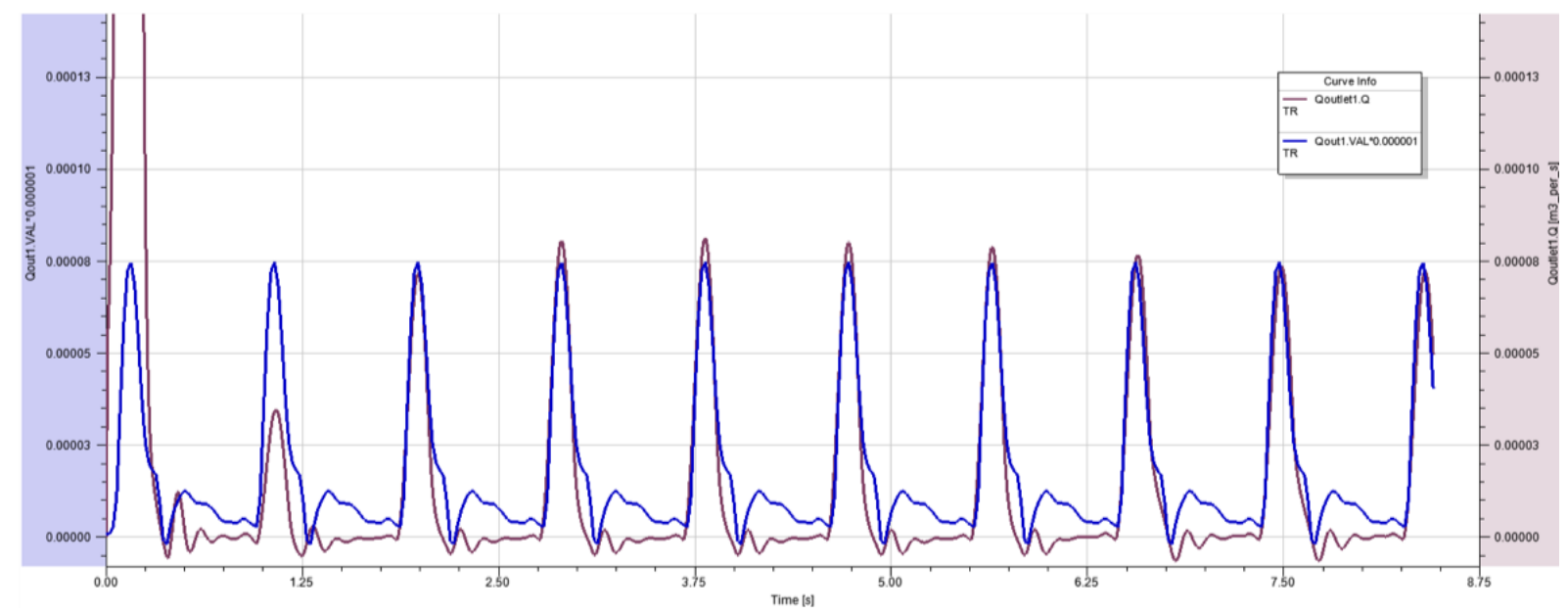

Figure 7: Comparison of flow curves from 2D PC MRI data (blue curve) and OD model simulations (burgundy curve)

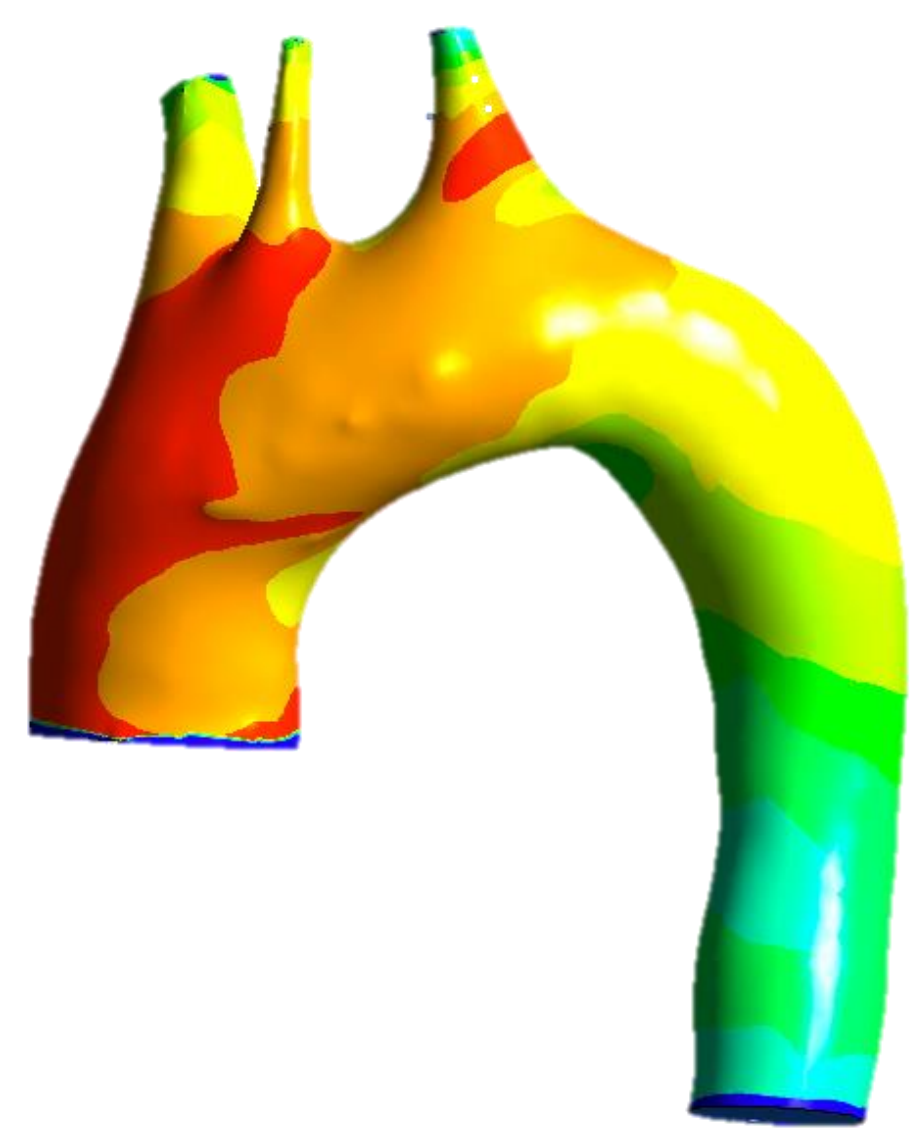

Figure 8: 3D representation of the aortic pressure wall according to simulation 


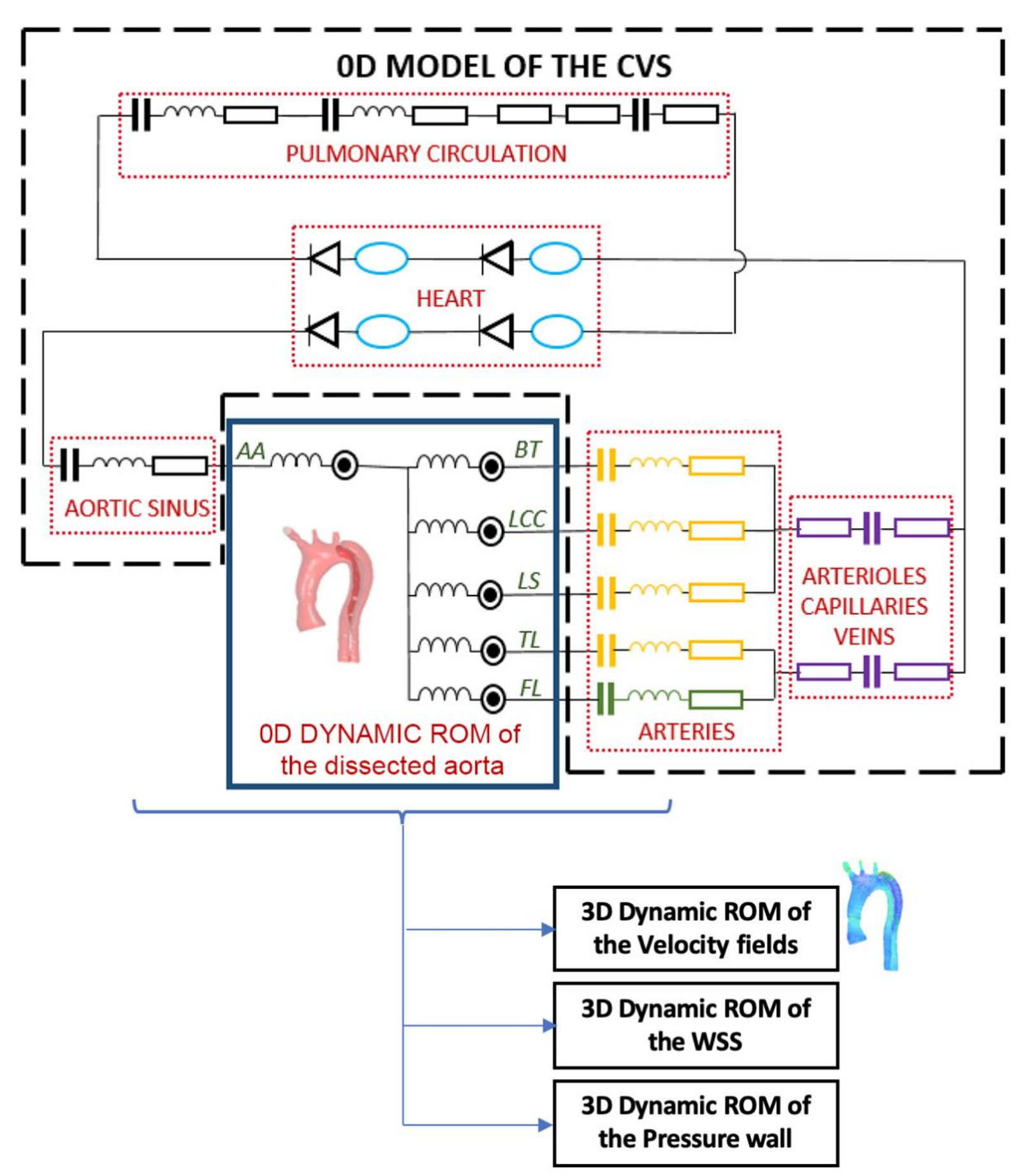

Figure 9: Final OD model 

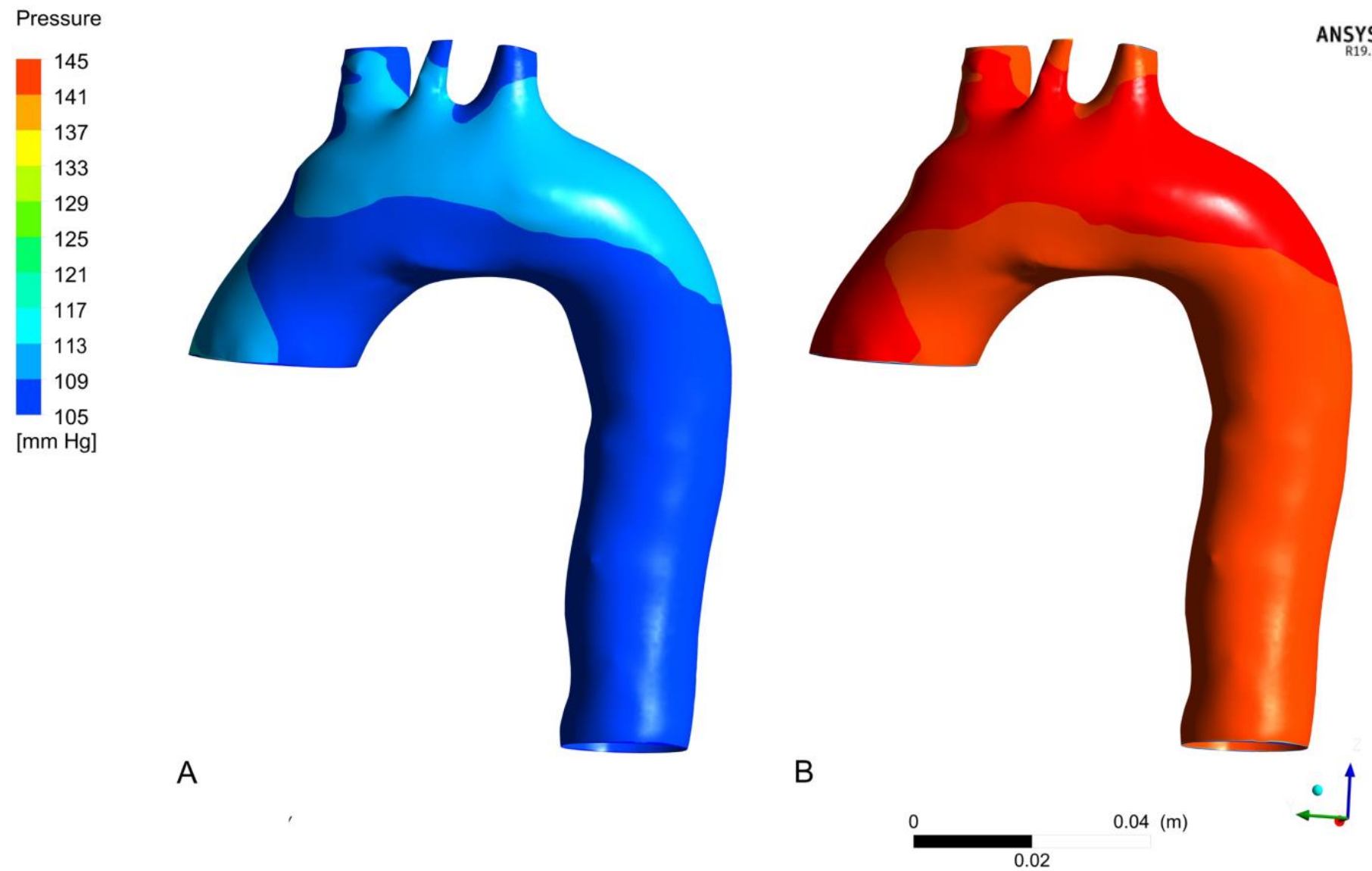

Figure 10: 3D modelling of aortic pressure wall of the healthy patient

A: from 2D PC MRI data, "controlled pressure" scenario

B: from OD model simulation, "high pressure" scenario 

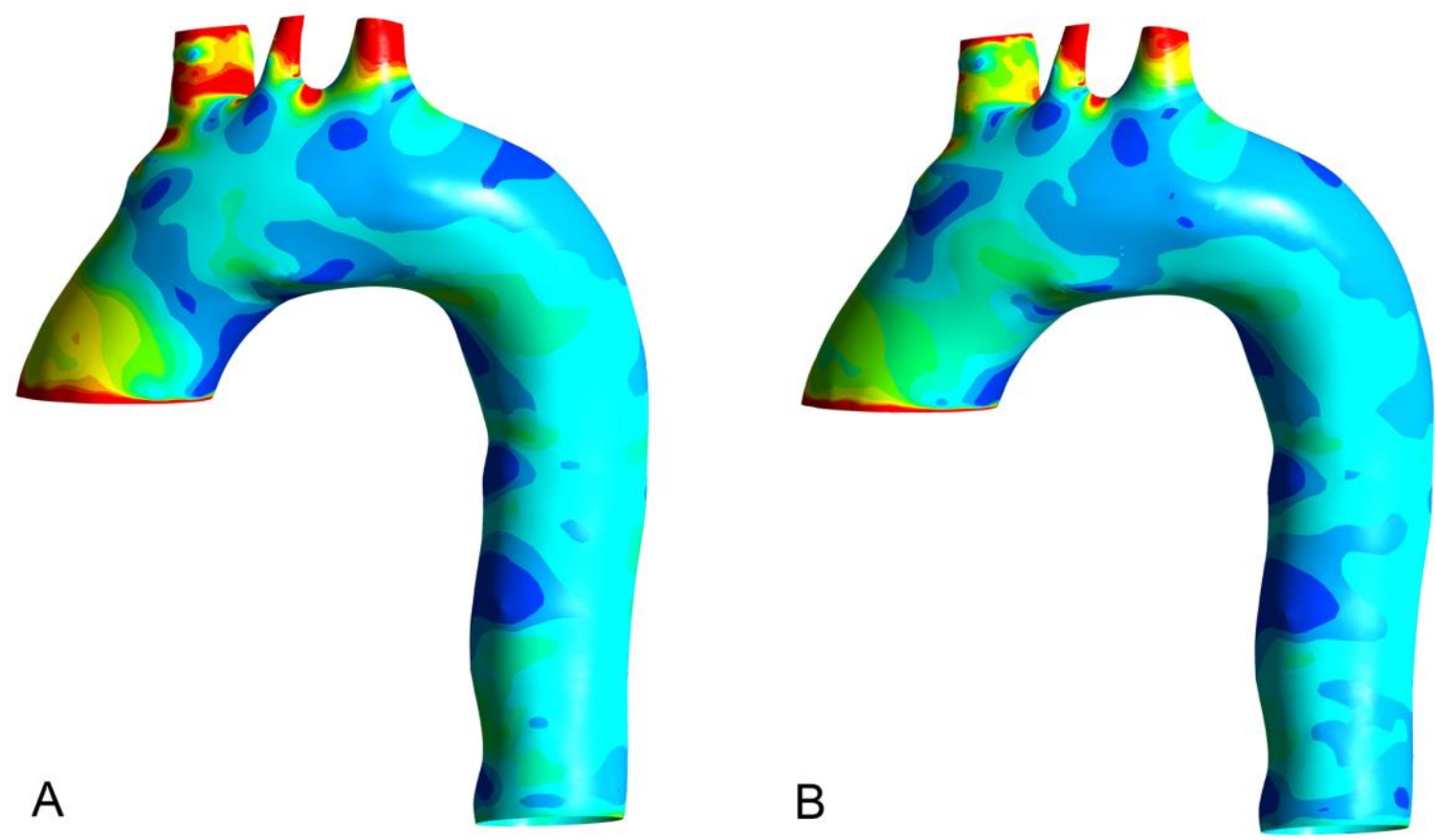

ANSYS

R19.1

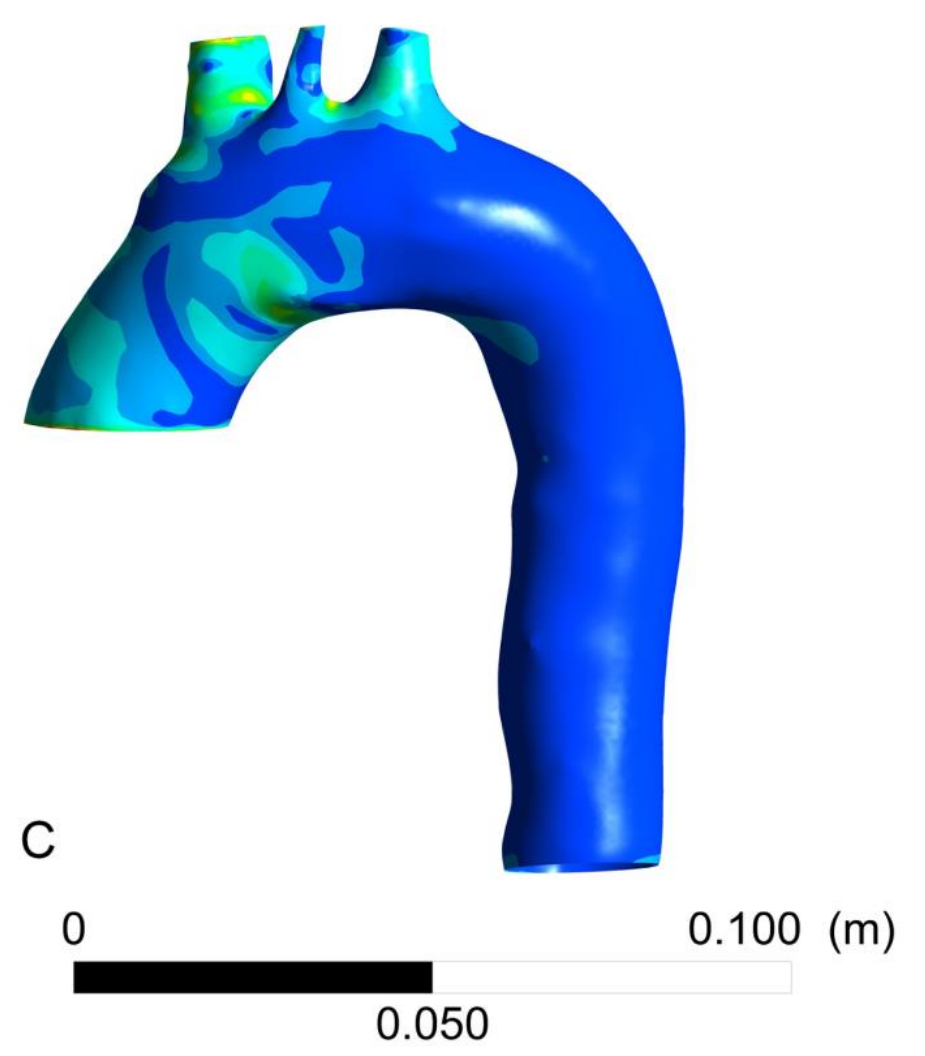

Figure 11: Comparison of 3D model of aortic wall shear stress of the healthy patient

A: 3D modeling of aortic wall shear stress of the healthy patient from 2D PC MRI data, "controlled pressure" scenario

B: 3D modeling of aortic wall shear stress of the healthy patient from OD model simulation,

"high pressure" scenario

C: Comparison of differences of aortic wall shear stress between the two scenarios 

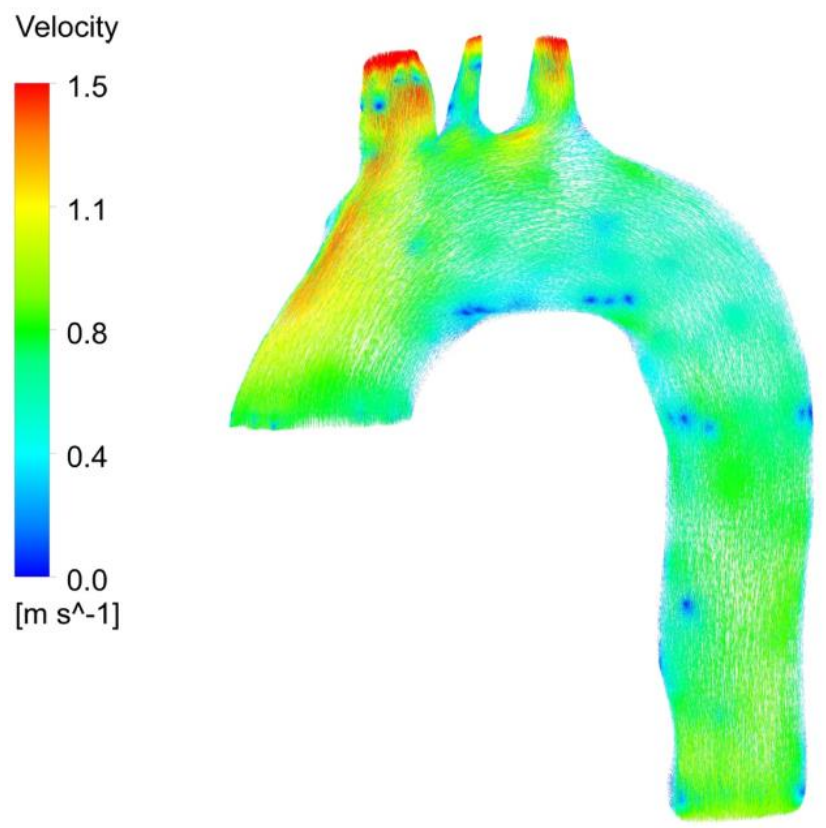

A

B

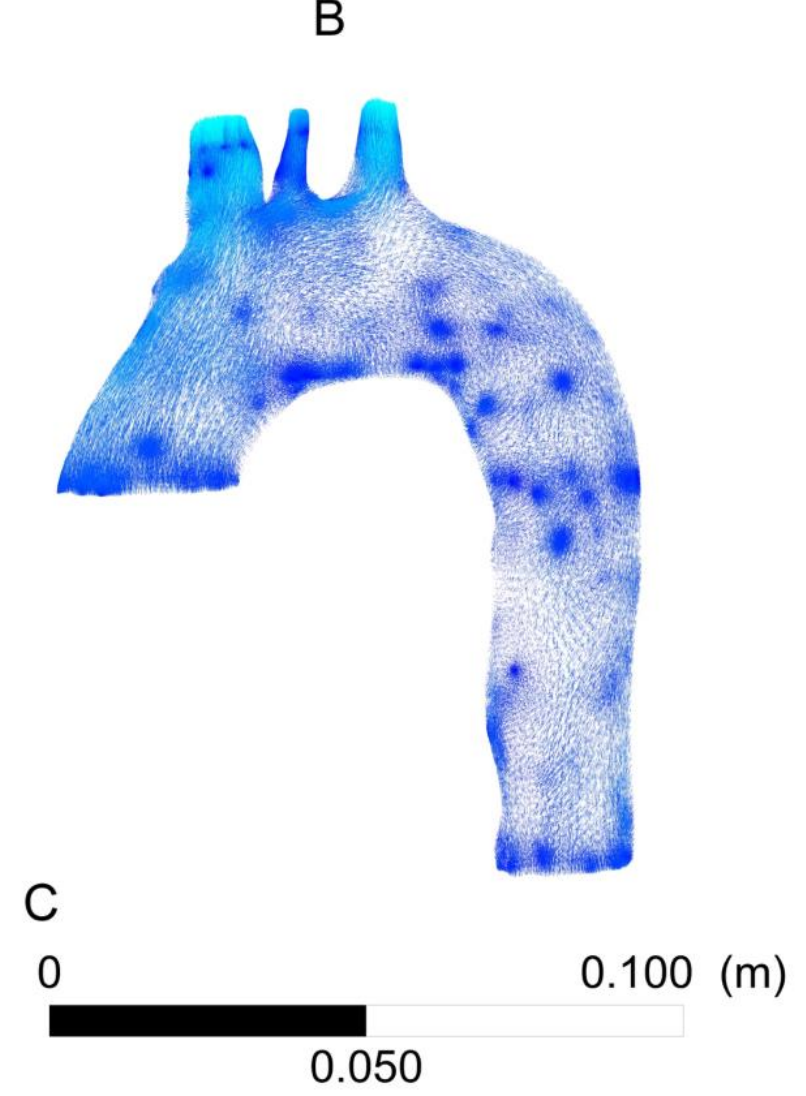

Figure 12: Comparison of 3D model of aortic blood velocities fields of the healthy patient

A: 3D modeling of velocities fields in the aorta of the healthy patient from 2D CP MRI data, "controlled pressure" scenario

B: 3D modeling of velocities fields in the aorta of the healthy patient from OD model simulation, "high pressure" scenario

C: Comparison of differences of velocities fields in the aorta between the two scenarios 
Pressure

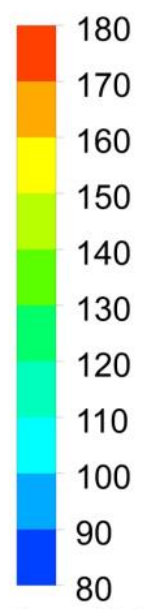
$[\mathrm{mm} \mathrm{Hg}]$
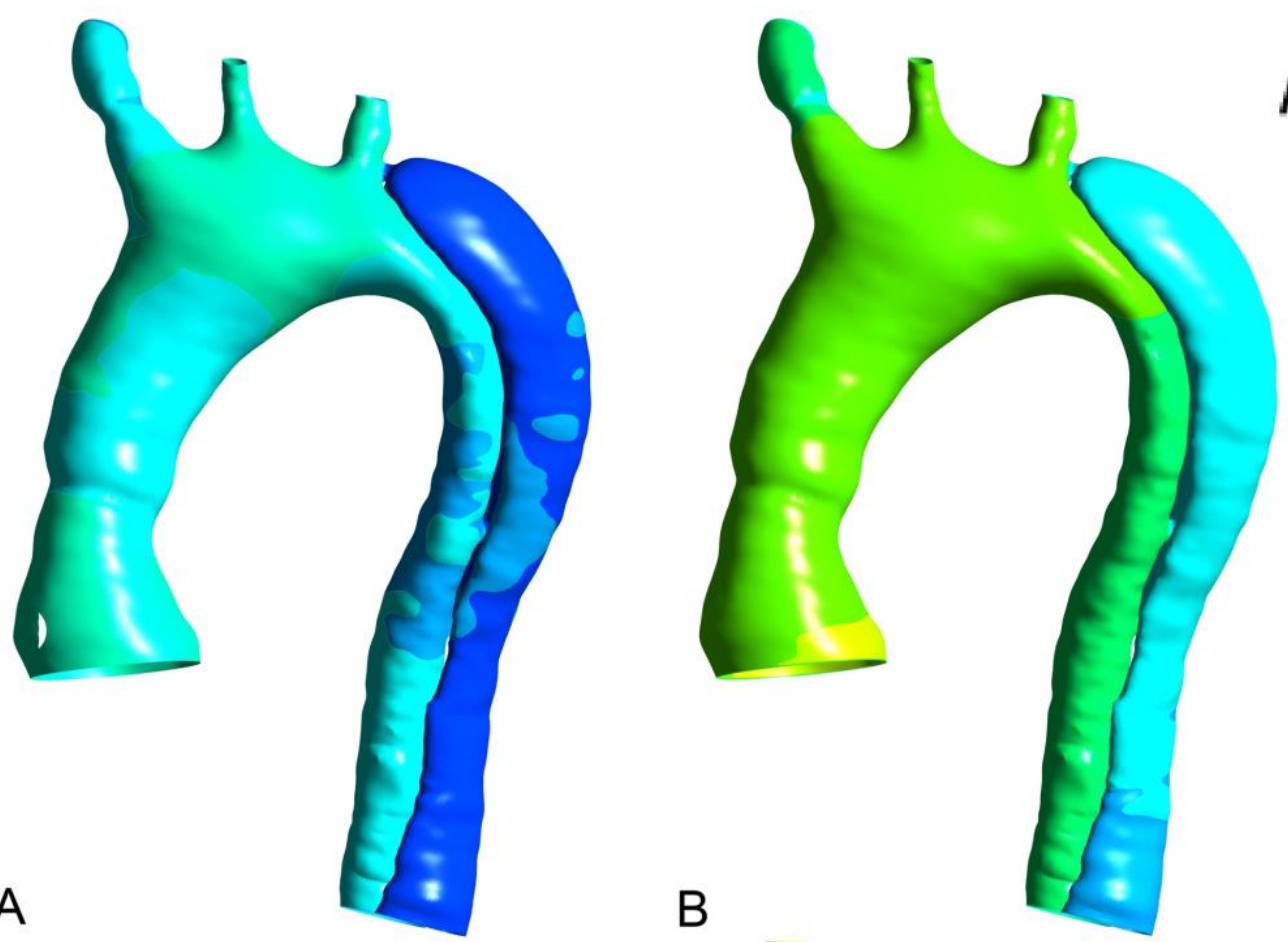

ANSYS

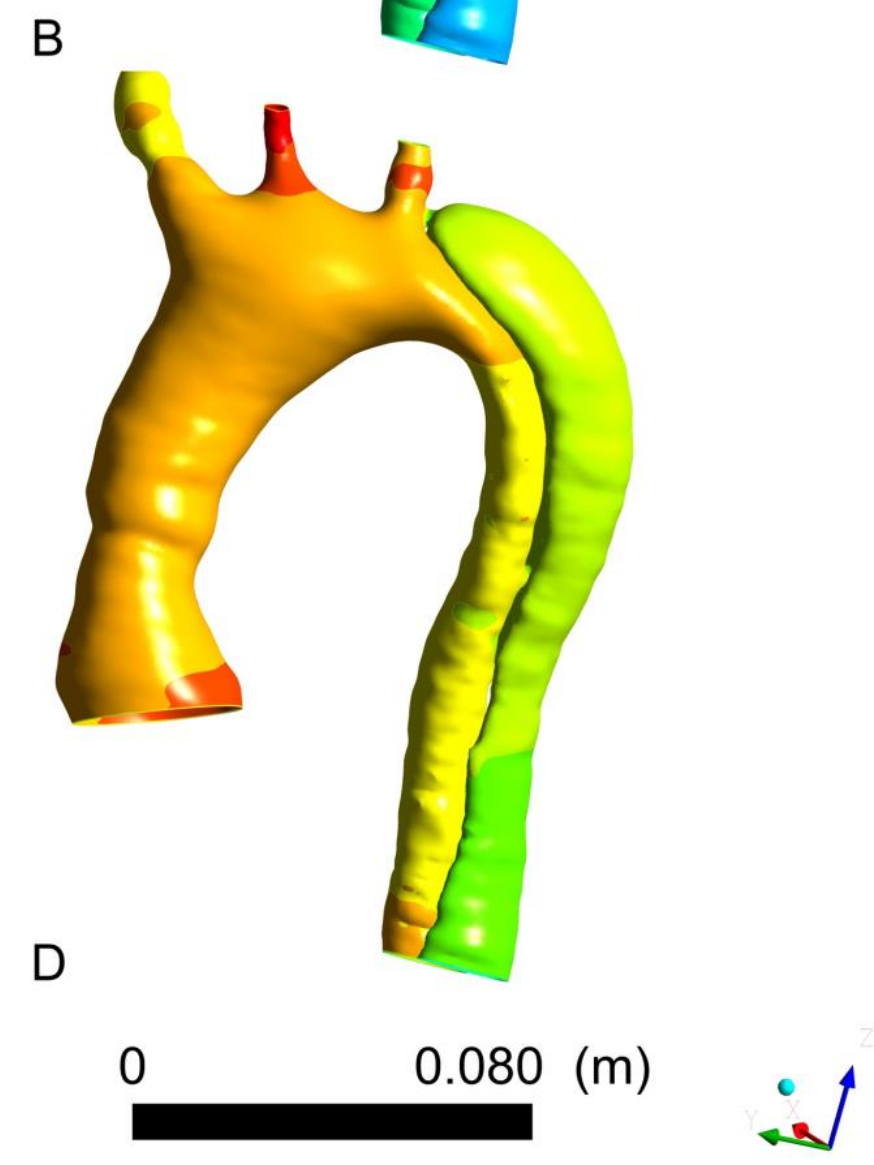

Figure 13: 3D modelling of aortic pressure wall of the dissected patient
A: from OD model simulation, "controlled pressure" scenario
B: from 2D CP MRI data, "moderately controlled pressure" scenario
C: from OD model simulation, "high pressure by vasoconstriction" scenario
D: from OD model simulation, "high pressure by hypervolemia " scenario 

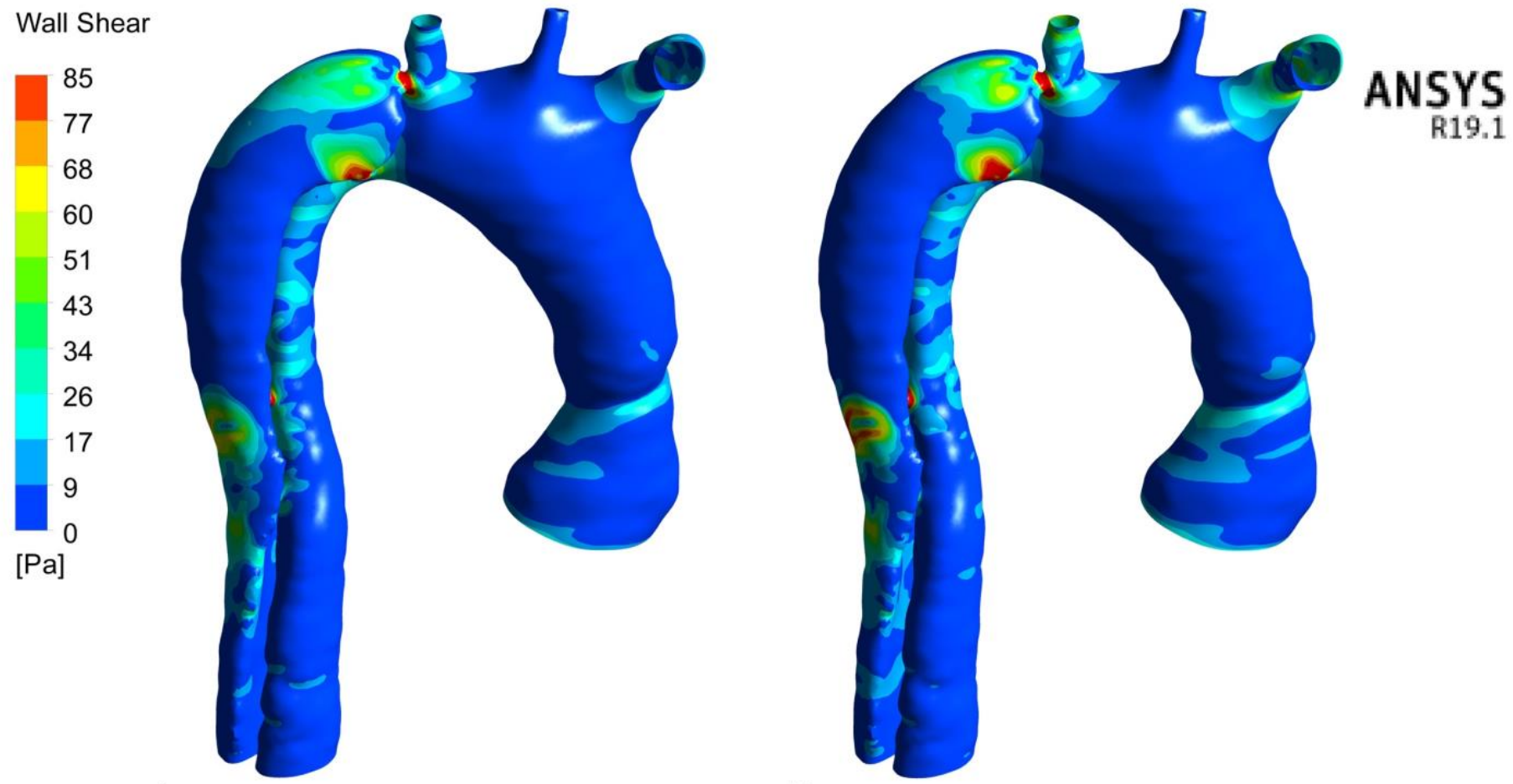

A

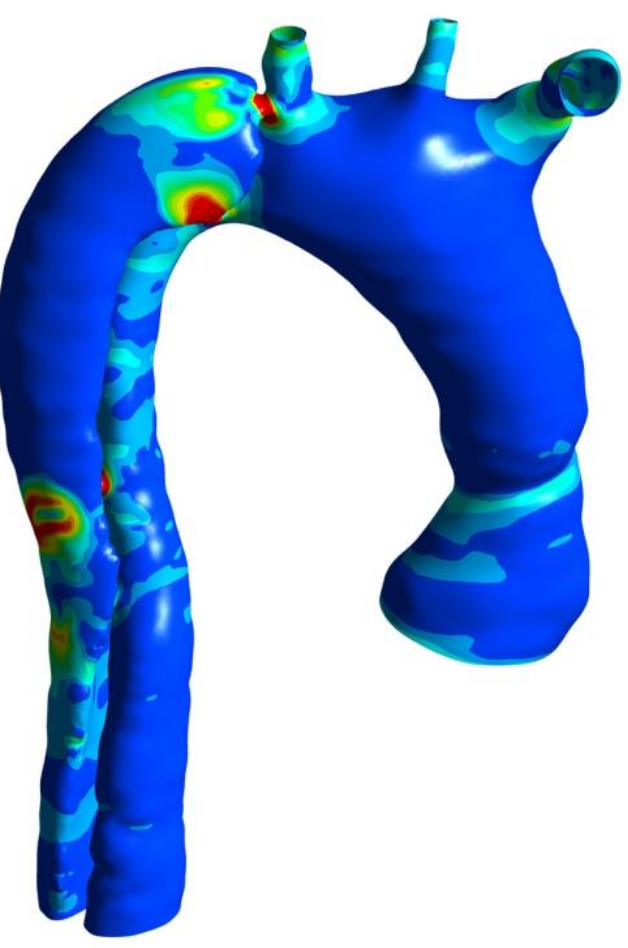

C

B

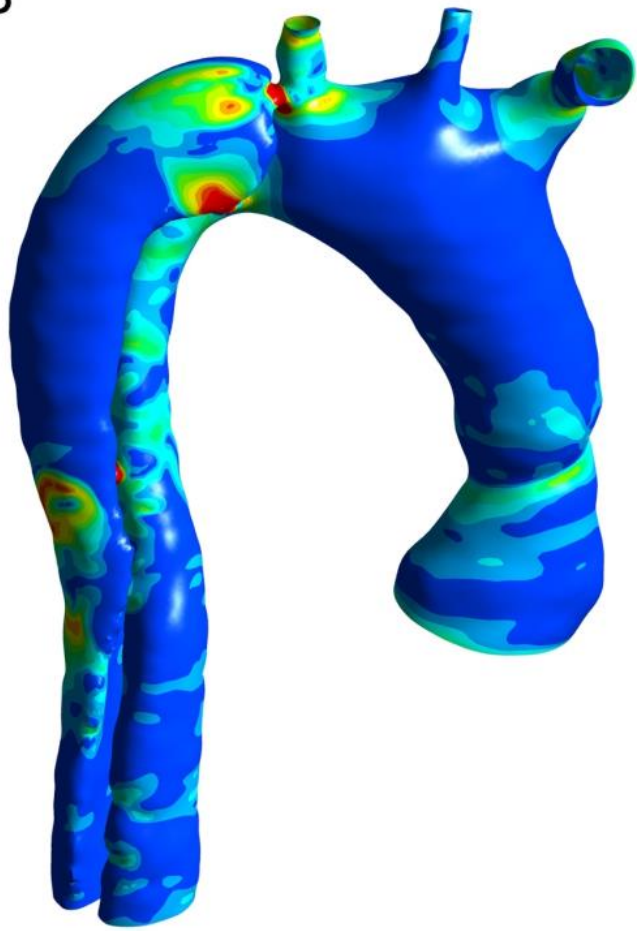

D

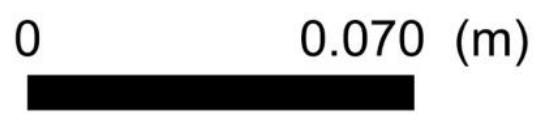

Figure 14: 3D Modeling of aortic wall shear stress of the dissected patient

A: from OD model simulation, "controlled pressure" scenario

B: from 2D CP MRI data, "moderately controlled pressure" scenario

C: from OD model simulation, "high pressure by vasoconstriction" scenario

D: from OD model simulation, "high pressure by hypervolemia " scenario 

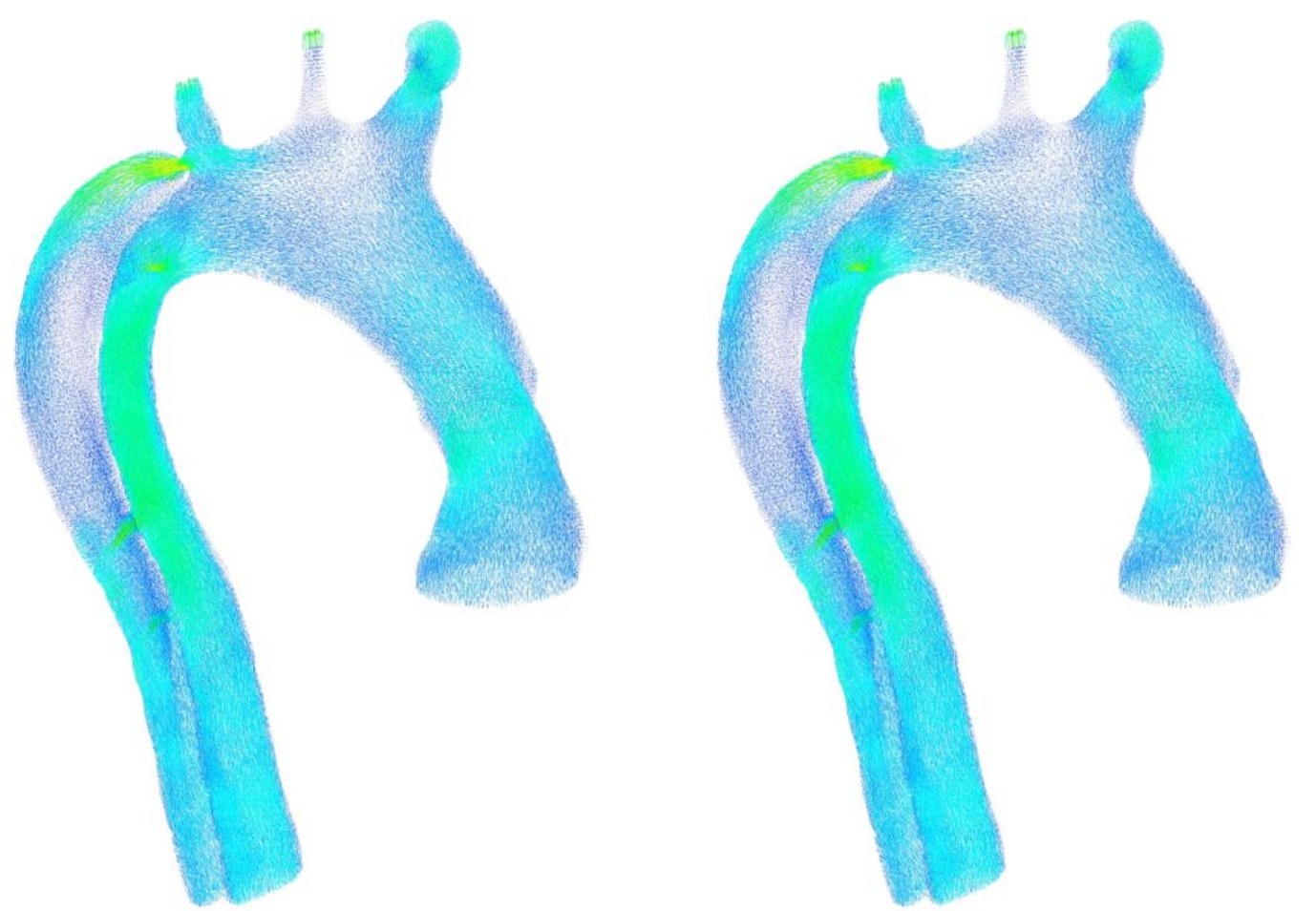

ANSYS

R19.1

A

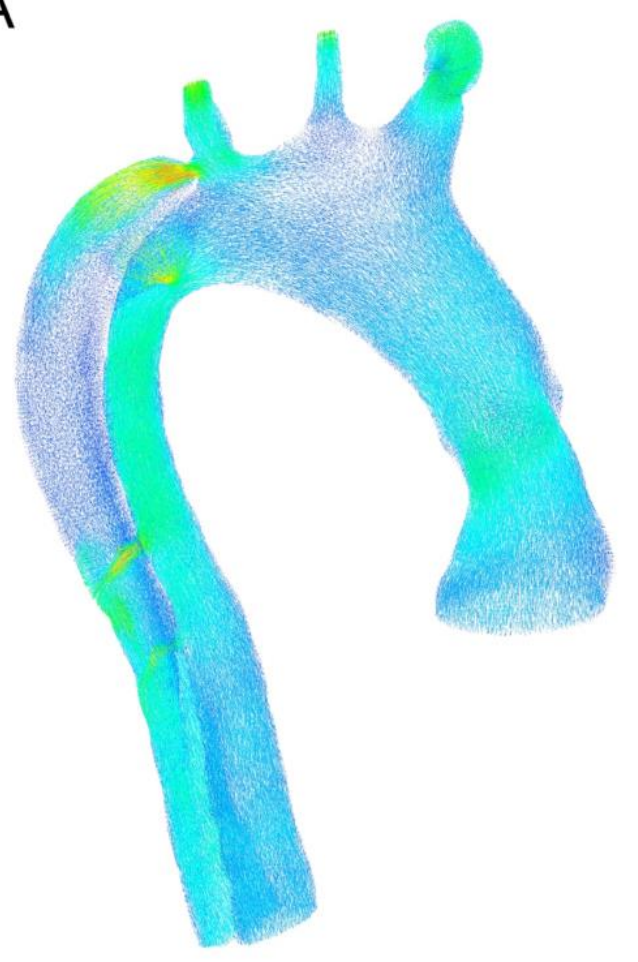

B

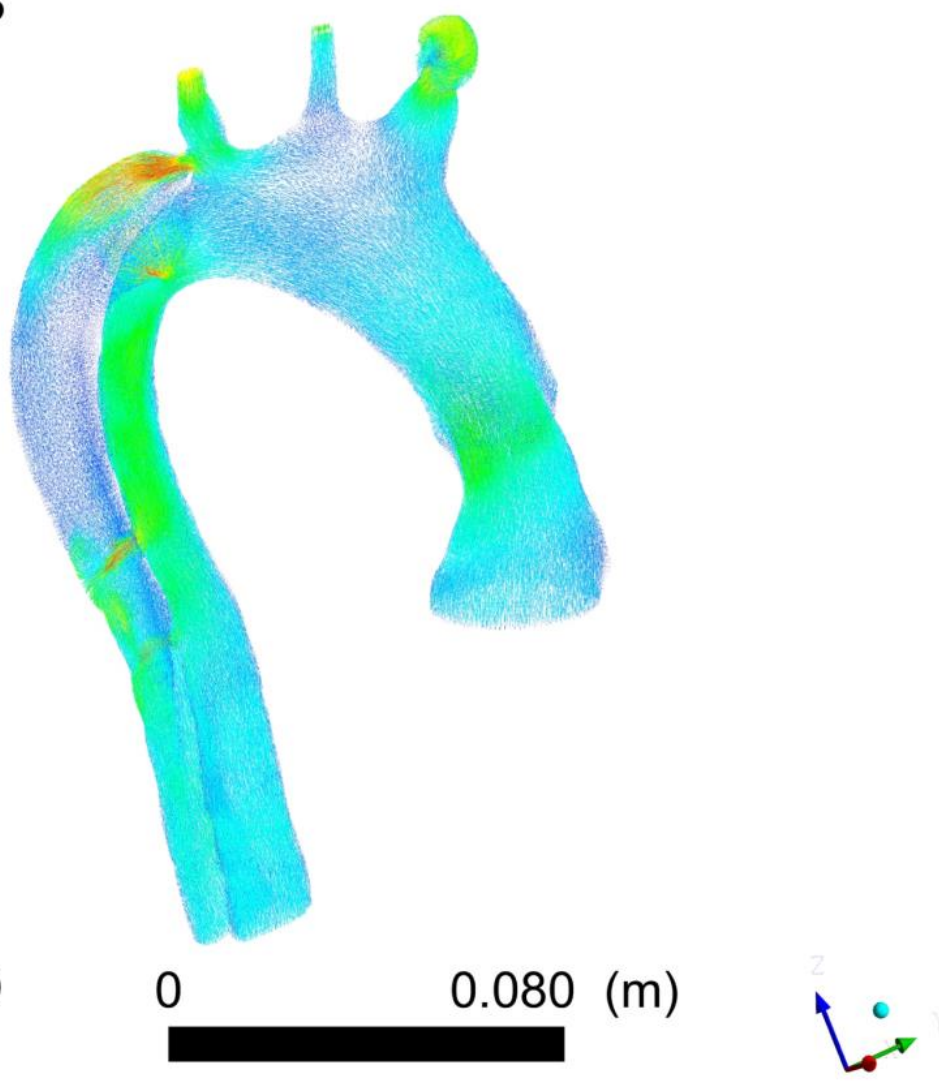

Figure 15: 3D modelling of aortic blood velocities field of the dissected patient

A: from OD model simulation, "controlled pressure" scenario

B: from 2D CP MRI data, "moderately controlled pressure" scenario

C: from OD model simulation, "high pressure by vasoconstriction" scenario

D: from OD model simulation, "high pressure by hypervolemia " scenario 